\title{
Wireless Injection Locking of Oscillator Circuits
}

\author{
Mabel Pontón, Member, IEEE, Almudena Suárez, Fellow, IEEE
}

\begin{abstract}
An in-depth investigation of the global behavior of wireless injection-locked oscillator circuits is presented. This kind of operation has been proposed for motion-sensing applications, in which each oscillator is also self-injection locked by the signal reflected by the target, with the overall system behaving in an autonomous manner. The analysis is based on a realistic description of the effect of the self-injection and mutual-injection signals, and the oscillator behavior, described with a reducedorder model, extracted from harmonic balance. As will be shown, sinusoidal dependences on the oscillation frequency, associated with the signal propagation, may give rise to turning points in the solution curves, whereas the mutual synchronization of the oscillator circuits inherently gives rise to a coexistence of solutions with different phase shifts. The investigation includes fundamental aspects such as the bifurcation phenomena and phase-noise variation with the distance and antenna gain. The aim is to develop a useful methodology for the efficient analysis and reliable prediction of the behavior of these promising systems. All the results obtained with the new formulation, of easy application, have been carefully validated with costly circuit level simulations of the whole system. For experimental validation, a prototype operating at $2.45 \mathrm{GHz}$ has been manufactured and measured.
\end{abstract}

Index Terms - Bifurcation, injection locking, phase noise, stability.

\section{INTRODUCTION}

$\mathrm{T}_{\mathrm{D}}^{\mathrm{H}}$ HE works [1-7] have demonstrated motion-sensing Doppler-radar systems for person localization and breathing-rate measurement. In [1] a ratio meter is used to compare the amplitudes of the reflected and reference signals, whereas [2]-[7] use mixers as phase detectors to compare the phases of the reflected and reference signals. On the other hand, the recent works [8-10] propose a compact implementation of the Doppler radar system, based on the self-injection of oscillator circuits [11]. In realizations based on a single oscillator [8-10], the oscillator signal is transmitted to the target, reflected and received with a certain phase

Manuscript received July 01, 2016; revised October 01, 2016. This work was supported by the Spanish Ministry of Economy and Competitiveness under the research project TEC2014-60283-C3-1-R and Juan de la Cierva Research Program IJCI-2014-19141 and by the Parliament of Cantabria under the project Cantabria Explora 12.JP02.64069. This paper is an expanded version from the IEEE International Microwave Symposium, San Francisco, CA, USA, May 22-27 2016. M. Pontón and A. Suárez are with the Departamento de Ingeniería de Comunicaciones, Universidad de Cantabria, Santander, 39005, Spain (e-mail: mabel.ponton@unican.es; almudena.suarez@unican.es). modulation, and the information is recovered with a delay-line demodulator [8]. The oscillator is locked to the reflected signal, so it operates as a self-injection-locked oscillator (SILO). As shown in [8-10], such a system has an uncertainty when detecting the response of a target with both inward/outward and back and forth motions, like the lungs of a person that is undesirably moving towards or away from the sensor. The recent works [12-13] propose a system to circumvent this problem, which is based on the use of two oscillator sensors that are mutually locked through wireless propagation. In this way, each of the two oscillator circuits is both self-injection locked by the signal reflected by the target and locked by the signal transmitted by the second oscillator. As shown in [12-13], this kind of operation enables a discrimination of the inward/outward and back and forth motions of the target.

Although the valid performance of these implementations has been experimentally demonstrated, the mathematical models are usually oversimplified. A direct application of Adler's equation [14] should not be accurate enough since both the single SILO and the two mutually locked SILOs behave in an autonomous manner, with synchronizing signals generated inside the system. In fact, the feedback in selfinjection-locked oscillators is a major mechanism for instability effects, as reported in the literature [15-16], and the stability analysis of mutually-locked SILOs has not been addressed yet.

In the previous work [17], a realistic formulation of a Doppler-radar sensor based on a single SILO was derived, using a reduced-order model of the oscillator circuit, extracted from harmonic-balance (HB), as shown in [18]. The formulation in [17] takes into account the nonlinear dependence of the SILO on the oscillation frequency. This derives from the phase shift between the reflected and transmitted signals (due to the signal propagation and reflection by the target), which is proportional to the oscillation frequency. Consideration of this dependence evidences the possible existence of multi-valued responses for some values of the antenna gain and distance to the target, which makes the stability essential [17].

Here a new formulation of the single SILO will be presented. Unlike the model in [17], which was based on a circulator, a model based on a voltage-controlled current source will be used. This is easier to implement and does not require any approximations of the reflected signal. It will be very convenient for the later analysis of the more complex 
system based on two mutually-locked SILOs. Since the stability, bifurcation and modulation analysis of the single oscillator system had been presented in [17], only the steadystate equation (with the new current- source model) and the phase-noise analysis, which was not tackled in [17], will be described here. Then, the case of two mutually-locked oscillators will be considered.

Each oscillator in the system of two mutually locked SILOs is both self-injection locked by the signal reflected by the target and locked by the signal transmitted by the second oscillator, with the overall system behaving in free-running conditions. As will be shown, the nonlinear dependence on the oscillation frequency leads to intricate solution curves and the mutual locking of the oscillator circuits gives rise to a coexistence of solutions with different phase shifts, as is inherent to the synchronized behavior. The investigation includes the fundamental aspects of stability, bifurcation analysis and phase noise. The phase modulation due to the target movement will also be addressed with an envelopetransient analysis, based on the new formulation.

The new methodologies and accompanying formulations have two main objectives. The first objective is the understanding of the complex-system behavior and its response when varying essential parameters, such as the distance to the target and the antenna gain. The second objective is the development of an accurate and easy-to-use analysis methodology, enabling a realistic prediction and optimization of the system behavior, which could be helpful for researcher in the topic.

All the formulations, providing the solution curves, stability properties, phase-noise and modulation effects, will be validated with costly harmonic-balance simulations, to demonstrate its accuracy. These simulations rely on the use of two auxiliary generators (AGs) [19-20]. Due to the multivalued nature of the solution curves, they are not able to provide these whole curves in all the cases, so some curve sections will be missing. Two prototypes operating at 2.45 $\mathrm{GHz}$ have been manufactured and measured, using several antennas with different gain values for a comparison as exhaustive as possible with the analysis results.

Section II presents the new formulation of the SILO including the phase-noise analysis. Section III describes the steady-state formulation of the system of mutually locked SILOS. Section IV and Section V, respectively, address the stability and phase-noise analysis of this system. Section VI presents the envelope-transient formulation, enabling the system analysis in modulated conditions.

\section{SELF-INJECTION LOCKED OSCILLATOR}

The SILO is shown in Fig. 1(a). The oscillator is loaded with an antenna that transmits the signal to the target, initially assumed motionless. The signal is reflected by the target and received back by the antenna, with a certain attenuation and phase shift. The transmitted power will be $\left|V_{\text {out }}\right|^{2} /\left(2 R_{r}\right)$, where $V_{\text {out }}$ is the oscillator output voltage. The received power depends on the transmitted power, the target radar section $\sigma$ and the propagation effects [3]. It will be expressed in terms of an equivalent injection-current source $I_{s}$, with the associated antenna conductance $1 / R_{r}$, connected at the output of the oscillator circuit, in the equivalent schematic of Fig. 1(b). For convenience, the oscillator phase origin at the fundamental output voltage, $\operatorname{ang}\left(V_{\text {out }}\right)=0$. In terms of the equivalent current source, the available power [21] at reception is:

$$
\frac{R_{r}\left|I_{s}\right|^{2}}{8}=\frac{V_{\text {out }}^{2}}{2 R_{r}} \frac{G_{t} G_{r} \sigma \lambda^{2}}{(4 \pi)^{3} d^{4}}
$$

where $G_{t}, G_{r}, d$ and $\lambda$ are, respectively, the transmitter and receiver antenna gains, the distance to the target and the wavelength at the SILO operation frequency $\omega_{s}$. Solving for $\left|I_{s}\right|$ one obtains:

$$
\left|I_{s}\right|=\frac{\lambda}{2 \pi R_{r} d^{2}} \sqrt{\frac{G_{t} G_{r} \sigma}{4 \pi}} V_{\text {out }}=\frac{c}{\omega_{s} R_{r} d^{2}} \sqrt{\frac{G_{t} G_{r} \sigma}{4 \pi}} V_{\text {out }}
$$

where $\sigma$ is the radar cross section (assumed $0.5 \mathrm{~m}^{2}$ ) and $c$ is the speed of light. For a distance to the target $d$, the round-trip phase shift should be: $-\omega_{s} 2 d / c$, and it will be possible to express the synchronizing signal as: $I_{s}=g\left(\omega_{s}, G_{\text {tot }}, d\right) V_{\text {out }}$, where the complex transconductance function $g$ is:

$$
g=\frac{c}{\omega_{s} R_{r} d^{2}} \sqrt{\frac{G_{t o t} \sigma}{4 \pi}} e^{-j \frac{2 d}{c} \omega_{s}}=\frac{\eta \sqrt{G_{\text {tot }}}}{\omega_{s} d^{2}} e^{-j \frac{2 d}{c} \omega_{s}}
$$

where $G_{\text {tot }}=G_{t} G_{r}$ is the total antenna gain and, for convenience, the following parameter has been defined:

$$
\eta=\frac{c}{2 R_{r}} \sqrt{\frac{\sigma}{\pi}}
$$

For antenna gains between $5 \mathrm{~dB}$ and $12 \mathrm{~dB}$, frequency $f_{s}=\omega_{s} /(2 \pi)=2.4 \mathrm{GHz}$ and distance $d$ in the order of 0.5 $\mathrm{m}$, the transconductance magnitude $\operatorname{mag}(g)$ is comprised between $1 \mathrm{mS}$ and $5 \mathrm{mS}$. Therefore, the amplitude of the synchronizing current $I_{s}$ should be small.

In free-running conditions, the total admittance function must be zero at any circuit node [22], and, in particular, at the output node. In the absence of the reflection signal, this output total admittance will be $Y_{T}\left(V_{o}, \omega_{o}\right)=0$. Note that when analyzing the oscillator circuit with an AG in HB [19-20], the condition $Y_{T}\left(V_{o}, \omega_{o}\right)=0$ constitutes an outer-tier equation, whereas the pure HB system, with all the harmonic content, constitutes the inner tier. In the presence of the synchronizing signal $I_{s}$ of small amplitude, connected in parallel at the oscillator output node [Fig. 1(b)], it will be possible to perform a Taylor-series expansion of the function $Y_{T}\left(V_{s}, \omega_{s}\right)$ about $V_{o}, \omega_{o}$, where $V_{s}, \omega_{s}$ are the amplitude and frequency in 
injection-locked conditions. Therefore, the steady-state oscillation condition is:

$Y_{T}\left(V_{s}, \omega_{s}\right) \cong Y_{V}\left(V_{s}-V_{o}\right)+Y_{\omega}\left(\omega_{s}-\omega_{o}\right)-g\left(\omega_{s}, G_{t o t}, d\right)=0$

where $Y_{V}$ and $Y_{\omega}$ stand for the amplitude and frequency derivatives of the admittance function at the free-running solution (without the reflected signal). For a realistic oscillator modeling, the derivatives $Y_{V}$ and $Y_{\omega}$ are calculated in a HB simulation of the standalone free-running oscillator, by applying finite differences to an AG [18,23]. Note that the pure $\mathrm{HB}$ system constitutes an inner tier for this derivative calculation. Splitting (5) into real and imaginary parts:

$$
\begin{aligned}
& G_{v}\left(V_{s}-V_{o}\right)+G_{\omega}\left(\omega_{s}-\omega_{o}\right)-\frac{\eta \sqrt{G_{\text {tot }}}}{\omega_{s} d^{2}} \cos \left(\omega_{s} \frac{2 d}{c}\right)=0 \\
& B_{v}\left(V_{s}-V_{o}\right)+B_{\omega}\left(\omega_{s}-\omega_{o}\right)+\frac{\eta \sqrt{G_{\text {tot }}}}{\omega_{s} d^{2}} \sin \left(\omega_{s} \frac{2 d}{c}\right)=0
\end{aligned}
$$

where the subindex indicates the variable with respect to which the derivative is calculated. Unlike the expression in [17], based on the use of an ideal circulator, system (6) does not involve a Taylor-series expansion in the model of the reflected signal. The new model is more accurate and considerably simpler, and will be very convenient for the analysis of the two mutually locked SILOs.

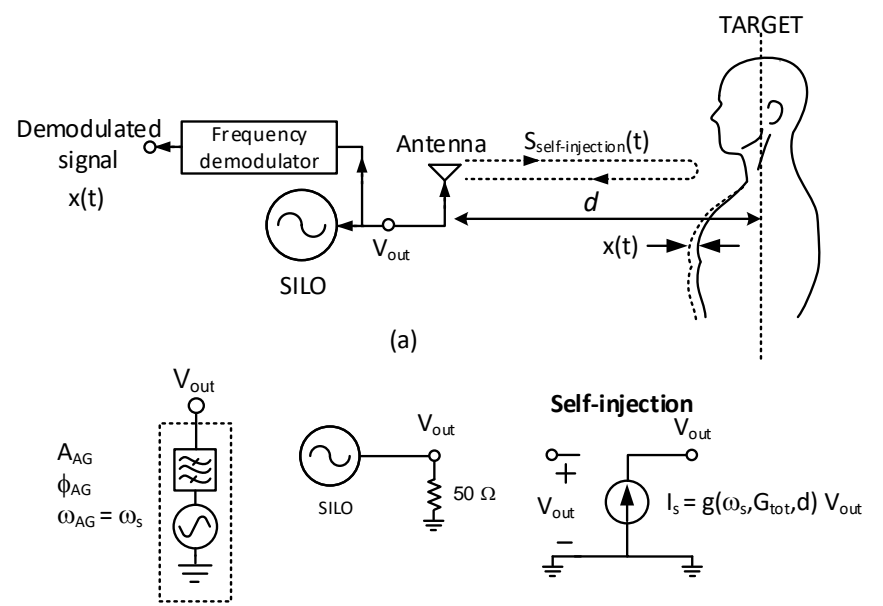

(b)

Fig. 1 Doppler radar system for motion sensing based on a SILO. (a) Operation with a single antenna. (b) Modeling of the self-injection effect with a voltage-controlled current source. For the HB simulations, an auxiliary generator (AG) is connected to the oscillator output node.

The double dependence on $\omega_{s}$ of (6), through both the oscillator-admittance linearization and the sinusoidal terms, will give rise to multi-valued solution curves for some values of the antenna gain $G_{\text {tot }}$ and distance to the target $d$. As gathered from (6), the impact of the sinusoidal terms will be stronger for higher $G_{\text {tot }}$. The existence of multi-valued sections will demand a stability analysis to distinguish between physical and unphysical solutions. This analysis, based on the application of a small amplitude perturbation to system (6), was presented in [17], together with conditions to detect the bifurcation points, at which there is a qualitative change of the stability properties.
The schematic and photograph of the SILO are shown in Fig. 2(a) and Fig. 2(b), respectively. The oscillator is based on the FET NE3210S01 and built on Rogers 4003C substrate. The variations of the oscillation frequency obtained with (6) for two antenna-gain values, $G=3.5 \mathrm{~dB}$ and $G=10 \mathrm{~dB}$, are shown in Fig. 3(a) and Fig. 3(b), respectively. Two different gain values are considered in order to test the influence of the antenna gain on the excursion of the oscillation frequency and the shape of the solution curves versus $d$. In agreement with the semi-analytical formulation, when increasing the antenna gain, the frequency excursion is larger and the solution curve becomes multi-valued in some $d$ intervals.

For the two gain values considered in Fig. 3, the solution curve obtained with (6) is compared with the one obtained with $\mathrm{HB}$, using the AG technique. The HB simulation is unable to provide the complete solution curve for the larger gain value $G=10 \mathrm{~dB}$ due to its multi-valued nature. Obtaining the missing sections would demand a cumbersome parameter switching between the AG variables and the actual parameter $d$, performed manually by the user [19-20]. The stable sections of the solution curves, obtained with the method in [17], have been represented with bolder lines. Measurements have been superimposed in the two cases with good agreement. Finally, Fig. 3(c) presents two coexisting stable spectra obtained experimentally for the same values of distance to the target, $d=0.451 \mathrm{~m}$, and antenna gain $G=10 \mathrm{~dB}$. This measurement demonstrates the possible coexistence of stable solutions for usual values of $d$ and $G$.

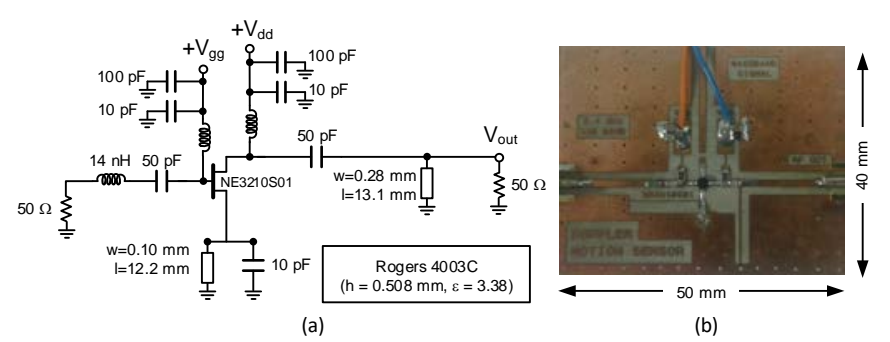

Fig. 2 Self-injection locked oscillator (SILO) at $2.45 \mathrm{GHz}$. (a) Schematic of the individual oscillator. (b) Photograph of the prototype built on Rogers 4003C substrate. 


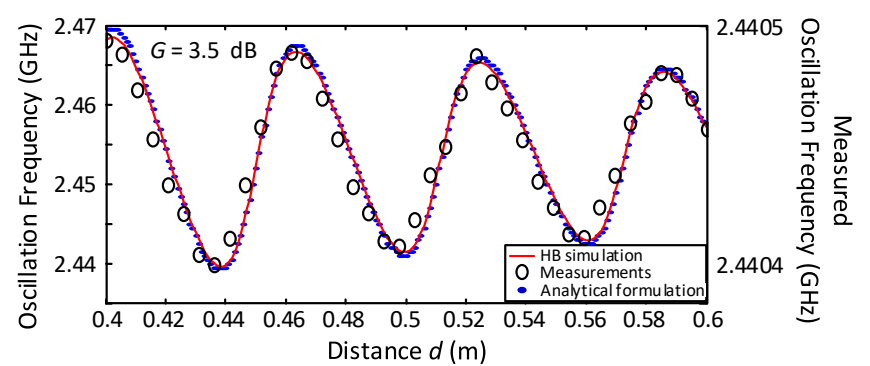

(a)

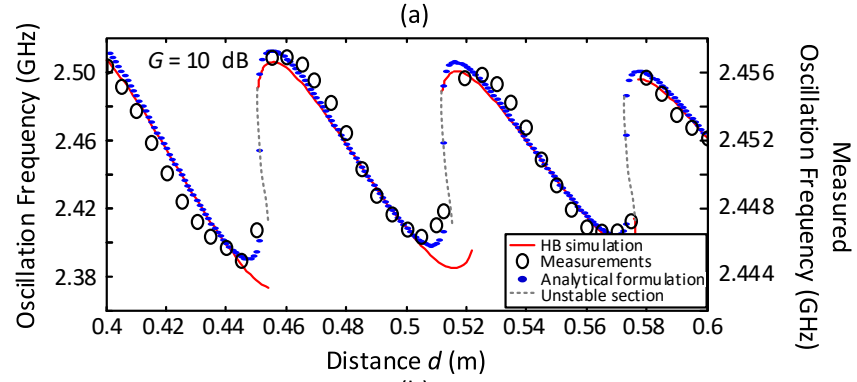

(b)

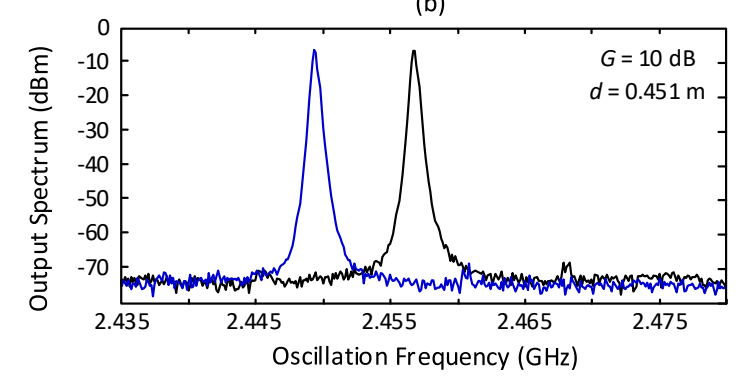

(c)

Fig. 3 Variation of the oscillation frequency with the distance to the target $d$ for two different values of antenna gain, also shown in Fig. 2 of [17]. (a) $G=3.5 \mathrm{~dB}$ and (b) $G=10 \mathrm{~dB}$. Results obtained with (6) are compared with those provided by HB with 8 harmonic terms.(c) Measured output spectra obtained for the same distance value $d=0.451 \mathrm{~m}$ and antenna gain $G=10$ $\mathrm{dB}$.

Next, the phase noise of the self-injection locked oscillator will be analyzed, since this characteristic had not been studied in [17]. For a better insight into the various dependences, only white noise sources will be initially considered. The analysis will be based on the extraction of an equivalent noise current source $I_{w}(t)$ that will be connected in parallel at the analysis node, the same one at which the equivalent synchronizing source $I_{S}$ is connected and the total admittance function $Y_{T}\left(V_{s}, \omega_{s}\right)$ is calculated. The method to extract this equivalent noise source from a HB-based phase-noise simulation of the original free-running oscillator is described in [24]. In the presence of the noise sources, the oscillator amplitude and phase will become $V_{s}+\delta v(t)$ and $\delta \phi(t)$, respectively, and the frequency will undergo a complex increment $j \omega_{s}+s$. For the noise analysis, a small $s$ can be assumed, so the total conductance and susceptance functions on the left-hand side of (6) can be expanded in a first-order Taylor series about $V_{s}, \omega_{s}$. The complex frequency $s$ will act like a time differentiator, which in the case of the oscillator admittance will give rise to the term $-j Y_{\omega}(\omega) \dot{\tilde{V}}(t)$, where
$\tilde{V}(t)=\left(V_{s}+\delta v(t)\right) e^{j \delta \phi(t)}$. An analogous term will arise from the frequency derivative of the transconductance, $g_{\omega}$, acting on $\tilde{V}(t-\tau)$, where $\tau=2 d / c$. The delay $\tau$ will give rise to an increment in the perturbed-system dimension, which, using the approach $\delta \phi(t-\tau) \cong \delta \phi(t)-\tau \delta \dot{\phi}(t)$, is formulated as:

$$
\begin{aligned}
& Y_{V} \delta v(t)+Y_{\omega}\left(-j \frac{\delta \dot{v}(t)}{V_{s}}+y(t)\right)- \\
& g_{\omega}\left(-j \frac{\delta \dot{v}(t)}{V_{s}}+y(t)-\tau \dot{y}(t)\right)=\frac{I_{w}(t)}{V_{s}} \\
& \delta \dot{\phi}(t)=y(t)
\end{aligned}
$$

However, for the delay corresponding to a distance of about $0.5 \mathrm{~m}$, in the order of $1 \mathrm{~ns}$, the product $\tau g_{\omega}$ will have a very small value, so one can neglect its effect on the noisy envelope. Note that this delay is relevant at the carrier frequency. The frequency derivative $g_{\omega}$ is given by:

$$
g_{\omega}=-\left(\frac{1}{\omega_{s}^{2}}+j \frac{\tau}{\omega_{s}}\right) \frac{c}{R_{r} d^{2}} \sqrt{\frac{G_{t o t} \sigma}{4 \pi}} e^{-j \tau \omega_{s}}
$$

After neglecting the effect of $\tau$ on the noisy envelope, splitting (7) into real and imaginary parts, one obtains:

$$
\begin{aligned}
& G_{v} \delta v(t)+T_{\omega}^{i} \frac{\delta \dot{v}(t)}{V_{s}}+T_{\omega}^{r} \delta \dot{\phi}(t)=\frac{I_{w}^{r}(t)}{V_{s}} \\
& B_{v} \delta v(t)-T_{\omega}^{r} \frac{\delta \dot{v}(t)}{V_{s}}+T_{\omega}^{i} \delta \dot{\phi}(t)=\frac{I_{w}^{i}(t)}{V_{s}}
\end{aligned}
$$

where the superindexes $r$ and $i$ indicate real and imaginary parts, respectively, and, for compactness, the following functions have been defined:

$$
\begin{aligned}
& T\left(j \omega_{s}, G_{t o t}, d\right)=Y_{\omega}\left(\omega_{s}-\omega_{o}\right)-g\left(\omega_{s}, G_{t o t}, d\right) \\
& T_{\omega}=Y_{\omega}-g_{\omega}
\end{aligned}
$$

Applying the Fourier transform, solving for $\delta \phi(\Omega)$, where $\Omega$ is the offset frequency, and multiplying by the complex conjugate $\delta \phi^{*}(\Omega)$ [24], one obtains the following expression for the phase-noise spectral density:

$$
|\delta \phi(\Omega)|^{2}=\frac{\left(\left|Y_{v}\right|^{2}+\frac{\left|T_{\omega}\right|^{2}}{V_{s}^{2}} \Omega^{2}\right) 2 \frac{\left|I_{w}(\Omega)\right|^{2}}{V_{s}^{2}}}{\left(G_{v} T_{\omega}^{i}-B_{v} T_{\omega}^{r}\right)^{2} \Omega^{2}+\Omega^{4} \frac{\left|T_{\omega}\right|^{4}}{V_{s}^{2}}}
$$

where it has been taken into account that $I_{w}^{r}(t)$ and $I_{w}^{i}(t)$ are uncorrelated and $\left|I_{w}^{r}(\Omega)\right|^{2}=\left|I_{w}^{i}(\Omega)\right|^{2}=2\left|I_{w}(\Omega)\right|^{2}$ [22]. Writing the function $T_{\omega}$ in terms of $\omega_{s}, G_{t o t}, d$, one obtains: 


$$
\begin{aligned}
& |\delta \phi(\Omega)|^{2}= \\
& \left(\Delta+\left[\left.Y_{v}\right|^{2}+\frac{\left|T_{\omega}\right|^{2}}{V_{s}^{2}} \Omega^{2}\right) 2 \frac{\left|I_{w}(\Omega)\right|^{2}}{V_{s}^{2}}\right. \\
& \left.\left.(\Delta) \sin \left(\frac{\omega_{s} 2 d}{c}\right)+U_{2}\left(\omega_{s}, d\right) \cos \left(\frac{\omega_{s} 2 d}{c}\right)\right]\right)^{2} \Omega^{2}+\Omega^{4} \frac{\left|T_{\omega}\right|^{4}}{V_{s}^{2}}
\end{aligned}
$$

where:

$$
\begin{aligned}
& U_{1}\left(\omega_{s}, d\right)=\eta \sqrt{G_{\text {tot }}}\left(-\frac{2}{c} \frac{B_{v}}{\omega_{s} d}-\frac{G_{v}}{\omega_{s}^{2} d^{2}}\right) \\
& U_{2}\left(\omega_{s}, d\right)=\eta \sqrt{G_{\text {tot }}}\left(\frac{2}{c} \frac{G_{v}}{\omega_{s} d}-\frac{B_{v}}{\omega_{s}^{2} d^{2}}\right)
\end{aligned}
$$

and $\Delta=G_{v} B_{\omega}-B_{v} G_{\omega}$ is the determinant of the original freerunning oscillator. From inspection of (13), the sinusoidal terms in the denominator of (12) will become more relevant at smaller values of the distance $d$. Local minima and maxima will also be observed due to the sinusoidal terms $\sin \left(\omega_{s} 2 d / c\right)$ and $\cos \left(\omega_{s} 2 d / c\right)$. The phase-noise behavior can be related with the stability properties of the SILO. As shown in [17], the reduced-order dynamical system contains two real eigenvalues. One is $\lambda_{1}=0$, due to the autonomous behavior of the free-running system. The second eigenvalue, $\lambda_{2}$, is negative (positive) in stable (unstable) sections of the solution curve, and equal to zero at the turning points clearly distinguished in the curves of Fig. 3. These turning points are given by the condition $\left(G_{v} T_{\omega}^{i}-B_{v} T_{\omega}^{r}\right)=0$. For low $\Omega$, the biggest contribution in the denominator of (12) corresponds to the term in $\Omega^{2}$. Since this term is directly proportional to $\left(G_{v} T_{\omega}^{i}-B_{v} T_{\omega}^{r}\right)$ [see (11)], the phase noise will significantly increase near the turning points, with the maxima belonging to the unstable sections. Due to the sinusoidal terms in the denominator, it is easily derived that the maxima are approximately spaced $\Delta d=c /\left(2 f_{s}\right)$, close to a half wavelength in free-running conditions. Note that the frequency $f_{s}$ varies with $d$. The maxima will be larger for smaller $\Omega$ due to a smaller contribution of the term in $\Omega^{4}$.

To consider the flicker noise, one would need an additional real equation, providing the circuit total conductance at baseband. The flicker noise will be modeled with a single current source, given by $I_{f}(t)$, connected at an internal circuit node, where this has a relevant effect [25]. The freerunning oscillator derivatives would be obtained using two AGs, one at dc and the other one at the fundamental frequency. Note that there is no need for the dc and firstharmonic equations of the reduced-order system to be defined at a same circuit node since each AG provides an outer tier equation and this can be introduced at arbitrary nodes. The phase-noise spectrum is obtained following a development analogous to the one carried out between (9) and (12). The resulting expressions are cumbersome and this is why they are omitted here. They also exhibit the double dependence on distance observed in (12).
The analysis has been applied to the system in Fig.1 and Fig. 2. The phase-noise spectrum obtained for antenna gain $G=10 \mathrm{~dB}$ and distance to the target $d=0.55 \mathrm{~m}$ is shown in Fig. 4, where it is compared with the experimental measurements. Unlike the case of the steady-state analysis in Fig. 3, it has not been possible to compare with HB simulations at circuit level, as the phase-noise simulation failed in all cases. Fig. 5 shows the variation of the phase noise spectral density at the constant offset frequency $f_{\text {off }}=100 \mathrm{kHz}$, through the solution curve in Fig. 3(b), traced versus $d$ and containing turning points. Measurements are superimposed with good agreement. The value of the minima and maxima depends on $d$, as evidenced by the dashed line superimposed in Fig. 5. The distance between both decreases with $d$, and for sufficiently large $d$ the spectrum should approximately agree with that of original (isolated) freerunning oscillator. As derived with (12), minima and maxima are approximately spaced half a wavelength. The narrow intervals with the highest phase-noise values correspond to the unstable regions of the solution curve in Fig. 3(b).

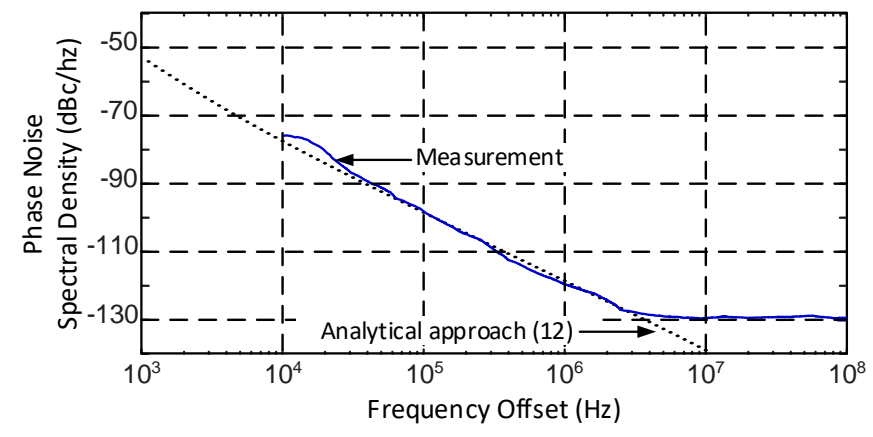

Fig. 4 Phase noise spectral density obtained with (12). Results of the analytical approach are compared with the experimental measurements.

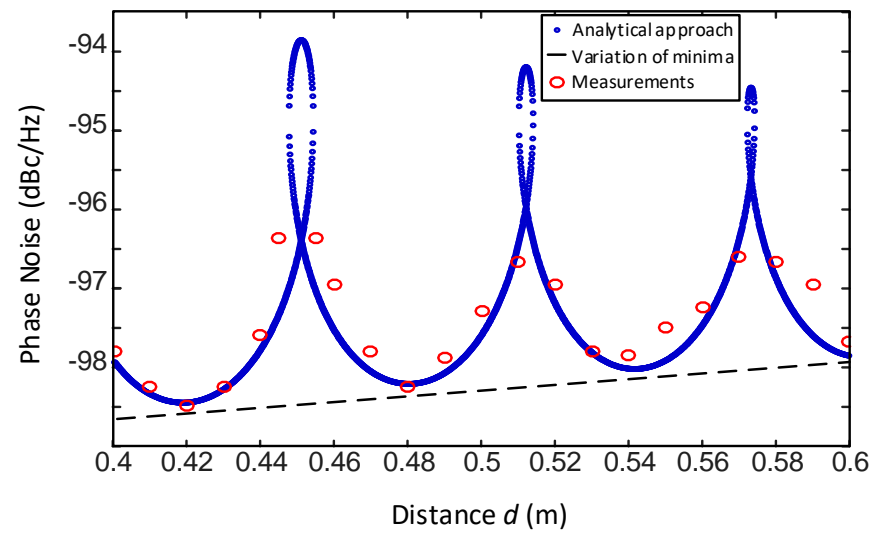

Fig. 5 Variation of the phase-noise spectral density with the distance $d$ at the constant offset frequency $f_{\text {off }}=100 \mathrm{kHz}$. The antenna gain is $G=10 \mathrm{~dB}$.

\section{MUtUAL INJECTION-LOCKING OF TWO SILOs}

As demonstrated in [12-13], the system in Fig. 6 enables a distinction between inward/outward and back and forth motions of the target. It is based on the use of two oscillators in a wireless injection-locking operation, so that each of the two oscillators is injection locked by the signal reflected by the target and by the signal transmitted by the second 
oscillator. The first oscillator ( $\operatorname{osc}_{1}$ ) is at a distance $d_{1}$ from the target and the second oscillator $\left(\mathrm{osc}_{2}\right)$ is at a distance $d_{2}$. The total distance between the oscillator elements is $d_{T}$.
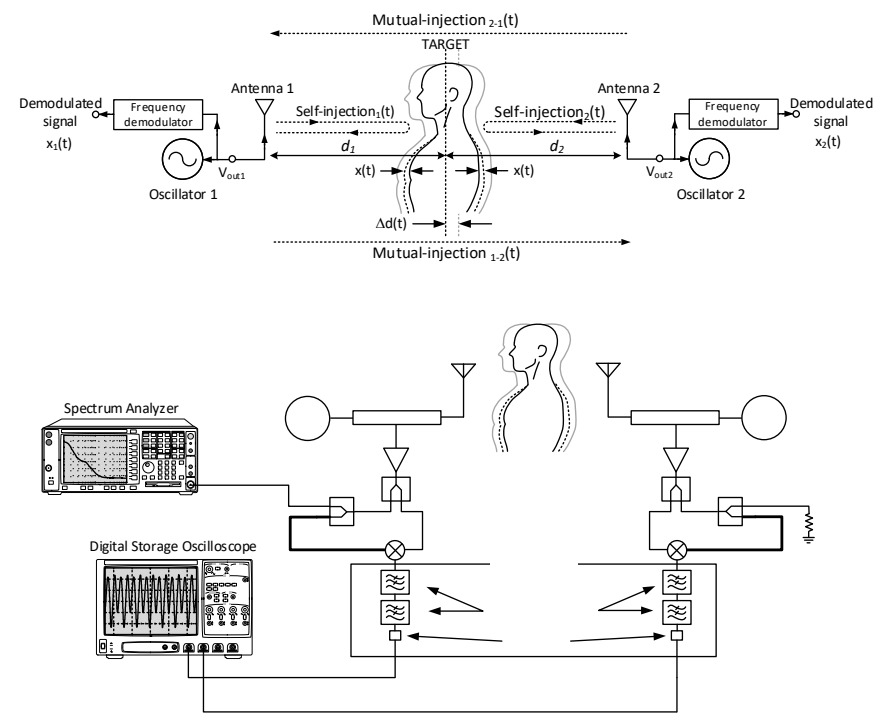

Fig. 6 Basic diagram of the mutual injection-locked system based on two selfinjection locked oscillators (SILOs). (a) Sketch of the complete system, with the definition of variables. (b) Measurement set-up.

Initially, the steady-state equations will be derived, considering a motionless target and assuming a synchronized behavior, so that the two oscillators operate at the same frequency $\omega_{s}$. Each oscillator is excited by two different injection signals, which will be modeled with voltagecontrolled current sources (Fig. 7). The self-injection transconductance functions corresponding to $\mathrm{osc}_{1}$ and $\mathrm{osc}_{2}$, and due to signal reflection by the target, are given by:

(14)

$$
\begin{aligned}
& g_{1}\left(\omega_{s}, G_{t o t 1}, d_{1}\right)=\frac{\eta \sqrt{G_{t o t}}}{\omega_{s} d_{1}^{2}} e^{-j \omega_{s} \frac{2 d_{1}}{c}}=D_{1} e^{-j \alpha_{1}}, \text { OsC }_{1} \\
& g_{2}\left(\omega_{s}, G_{t o t 2}, d_{2}\right)=\frac{\eta \sqrt{G_{\text {tot } 2}}}{\omega_{s} d_{2}^{2}} e^{-j \omega_{s} \frac{2 d_{2}}{c}}=D_{2} e^{-j \alpha_{2}}, \text { osC }_{2}
\end{aligned}
$$

where the magnitudes of the transconductances have been renamed as $D_{1}$ and $D_{2}$ and the corresponding phase shifts have been renamed as $-\alpha_{1},-\alpha_{2}$ for compactness. On the other hand, the signal transmitted by osc $\mathrm{osc}_{2}$ ) and received by osc $_{1}\left(\mathrm{osc}_{2}\right)$ constitutes a second synchronizing signal for this oscillator. This synchronizing signal will also be modeled as voltage-controlled current source (Fig. 7). In each case, the control voltage corresponds to the output voltage of the opposite oscillator circuit. The corresponding synchronizing currents $I_{m 1}$ (injecting ${ }^{\circ c_{1}}$ ) and $I_{m 2}$ (injecting osc $_{2}$ ) are:

$$
\begin{aligned}
& \operatorname{osc}_{1}: I_{m 1}=\frac{c \sqrt{G_{\text {tot } 1} G_{\text {tot } 2}}}{\omega_{s} R_{r}\left(d_{1}+d_{2}\right)} e^{-j \omega_{s} \frac{d_{T}}{c}} V_{\text {out } 2}=A e^{-j \beta} V_{\text {out } 2} \\
& \mathrm{osC}_{2}: I_{m 2}=\frac{c \sqrt{G_{\text {tot } 1} G_{\text {tot } 2}}}{\omega_{s} R_{r}\left(d_{1}+d_{2}\right)} e^{-j \omega_{s} \frac{d_{T}}{c}} V_{\text {out } 1}=A e^{-j \beta} V_{\text {out } 1}
\end{aligned}
$$

where $V_{\text {out } 1}, V_{\text {out } 2}$ are the output-voltage phasors of the two oscillator elements. The two transconductance functions associated with the mutual synchronization are identical and given by $A e^{-j \beta}$. Because of the synchronized operation of the two distinct oscillator circuits, there will be a time-constant phase shift $\phi$ between the two oscillator signals. Taking the phase origin at the output of the first oscillator, one can write:

$$
\begin{aligned}
& V_{\text {out } 1}=V_{1} \\
& V_{\text {out } 2}=V_{2} e^{j \phi}
\end{aligned}
$$

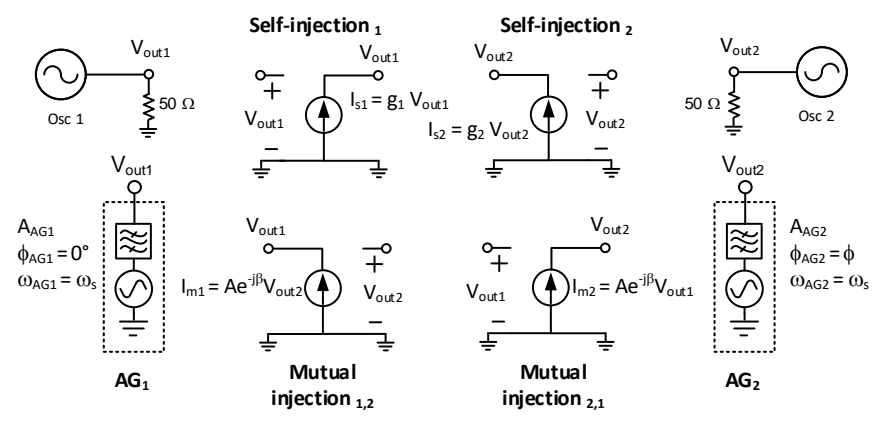

Fig. 7 Self-injection and mutual-injection effects have been modeled using voltage controlled current sources. For the HB simulations, two AGs have been used, each connected to one of the two oscillator circuits.

In the general case, the addition of the two injection currents will have small amplitude. Then, it will be possible to expand its corresponding admittance function in a Taylor series about the free-running solution, given by the amplitude and frequency $V_{o 1}, \omega_{o 1}$, for osc 1 , and by $V_{o 2}, \omega_{o 2}$, for osc2. The Taylor-series expansions provide:

$$
\begin{aligned}
& Y_{1 v}\left(V_{1}-V_{o 1}\right)+Y_{1 \omega}\left(\omega_{s}-\omega_{o 1}\right)= \\
& D_{1} e^{-j \alpha_{1}}+A \frac{V_{o 2}}{V_{o 1}} e^{-j \beta} e^{j \phi}=D_{1} e^{-j \alpha_{1}}+C_{1} e^{-j \beta} e^{j \phi}=0 \\
& Y_{2 v}\left(V_{2}-V_{o 2}\right)+Y_{2 \omega}\left(\omega_{s}-\omega_{o 2}\right)= \\
& D_{2} e^{-j \alpha_{2}}+A \frac{V_{o 1}}{V_{o 2}} e^{-j \beta} e^{-j \phi}=D_{2} e^{-j \alpha_{2}}+C_{2} e^{-j \beta} e^{-j \phi}=0
\end{aligned}
$$

where the new functions $C_{1}$ and $C_{2}$ have been introduced for convenience and the subindex $s$ (indicating a steady-state synchronized solution) has been dropped, for simplicity, in the oscillation amplitudes. To solve (17), each equation it will be decomposed into real and imaginary parts:

$$
\begin{aligned}
& \left.\begin{array}{l}
G_{1 v} \Delta V_{1}+G_{1 \omega} \Delta \omega_{1}=D_{1} \cos \alpha_{1}+C_{1} \cos (\phi-\beta) \\
B_{1 v} \Delta V_{1}+B_{1 \omega} \Delta \omega_{1}=-D_{1} \sin \alpha_{1}+C_{1} \sin (\phi-\beta)
\end{array}\right\} S_{s 1} \\
& \left.\begin{array}{l}
G_{2 v} \Delta V_{2}+G_{2 \omega} \Delta \omega_{2}=D_{2} \cos \alpha_{2}+C_{2} \cos (\phi+\beta) \\
B_{2 v} \Delta V_{2}+B_{2 \omega} \Delta \omega_{2}=-D_{2} \sin \alpha_{2}-C_{2} \sin (\phi+\beta)
\end{array}\right\} S_{s 2}
\end{aligned}
$$

where the increments $\Delta V_{1}=V_{1}-V_{o 1}, \quad \Delta V_{2}=V_{2}-V_{o 2}$, $\Delta \omega_{1}=\omega_{s}-\omega_{o 1}, \quad \Delta \omega_{2}=\omega_{s}-\omega_{o 2}$ have been defined. Then, subsystem $S_{s 1}$ and $S_{s 2}$ are solved for $\Delta \omega_{1}$ and $\Delta \omega_{2}$, which provides: 


$$
\begin{aligned}
& \omega_{s}=\omega_{o 1}-\frac{D_{1} G_{1 v}}{\Delta_{1}} \sin \alpha_{1}+\frac{C_{1} G_{1 v}}{\Delta_{1}} \sin (\phi-\beta)- \\
& \frac{D_{1} B_{1 v}}{\Delta_{1}} \cos \alpha_{1}-\frac{C_{1} B_{1 v}}{\Delta_{1}} \cos (\phi-\beta)
\end{aligned}
$$

where $\Delta_{1}=G_{1 v} B_{1 \omega}-B_{1 v} G_{1 \omega}$, and:

$$
\begin{aligned}
& \omega_{s}=\omega_{o 2}-\frac{D_{2} G_{2 v}}{\Delta_{2}} \sin \alpha_{2}-\frac{C_{2} G_{2 v}}{\Delta_{2}} \sin (\phi+\beta)- \\
& \frac{D_{2} B_{2 v}}{\Delta_{2}} \cos \alpha_{2}-\frac{C_{2} B_{2 v}}{\Delta_{2}} \cos (\phi+\beta)
\end{aligned}
$$

where $\Delta_{2}=G_{2 v} B_{2 \omega}-B_{2 v} G_{2 \omega}$. Expanding the cosine and sine functions (19) and (20), and taking into account that the unknown synchronization frequency $\omega_{\mathrm{s}}$ must be the same in the two real equations, one obtains a linear system of two real equations in the unknowns $\sin \phi, \cos \phi$. Valid solutions will fulfill: $\sin ^{2} \phi+\cos ^{2} \phi=1$, which will be the criterion for the numerical calculation of the solution curves versus any system parameter. Once $\sin \phi, \cos \phi$ have been calculated, the phase shift between the two oscillators is obtained directly.

If the two oscillator sub-systems are identical, with equal antenna gains and distances to the target, the two equations (18) and (20) will simplify to:

$$
\begin{aligned}
& \omega_{s}=\omega_{o}-\frac{D_{1} G_{1 v}}{\Delta_{1}} \sin \alpha_{1}+\frac{C_{1} G_{1 v}}{\Delta_{1}} \sin (\phi-\beta)- \\
& \frac{D_{1} B_{1 v}}{\Delta_{1}} \cos \alpha_{1}-\frac{C_{1} B_{1 v}}{\Delta_{1}} \cos (\phi-\beta) \\
& \omega_{s}=\omega_{o}-\frac{D_{1} G_{1 v}}{\Delta_{1}} \sin \alpha_{1}-\frac{C_{1} G_{1 v}}{\Delta_{1}} \sin (\phi+\beta)- \\
& \frac{D_{1} B_{1 v}}{\Delta_{1}} \cos \alpha_{1}-\frac{C_{1} B_{1 v}}{\Delta_{1}} \cos (\phi+\beta)
\end{aligned}
$$

where $\omega_{o}$ is the common free-running oscillation frequency. Because both equations should provide the same frequency $\omega_{s}$ , there will exist two phase solutions: $\phi=0^{\circ}$ and $\phi=180^{\circ}$, for each $\omega_{s}$. For each phase shift, the SILO frequency is:

$$
\begin{aligned}
& \phi=0^{\circ} \text { solution: } \\
& \omega_{s}=\omega_{o}-\frac{D_{1} G_{1 v}}{\Delta_{1}} \sin \alpha_{1}-\frac{C_{1} G_{1 v}}{\Delta_{1}} \sin (\beta)- \\
& \frac{D_{1} B_{1 v}}{\Delta_{1}} \cos \alpha_{1}-\frac{C_{1} B_{1 v}}{\Delta_{1}} \cos (\beta) \\
& \phi=180^{\circ} \text { solution: } \\
& \omega_{s}=\omega_{o}-\frac{D_{1} G_{1 v}}{\Delta_{1}} \sin \alpha_{1}+\frac{C_{1} G_{1 v}}{\Delta_{1}} \sin (\beta)- \\
& \frac{D_{1} B_{1 v}}{\Delta_{1}} \cos \alpha_{1}+\frac{C_{1} B_{1 v}}{\Delta_{1}} \cos (\beta)
\end{aligned}
$$

The above analysis method has been applied to the system in Fig. 6, using the oscillators in Fig. 2. Initially, identical oscillators, antenna gain, $G=3.5 \mathrm{~dB}$, and distance to the target $d_{1}=d_{2}$ have been considered. Fig. 8(a) shows the variation of the oscillation frequency for the in-phase and $180^{\circ}$ solution, versus the distance $d_{1}=d_{2}$. The two solution curves exhibit an oscillatory behavior about the free-running frequency $\omega_{0}$. The excursion between the minima and maxima will approach zero for a sufficiently large $d$, with the two oscillators tending to their original free-running behavior (assumed equal).

For comparison, the same analysis has been carried at circuit-level, using HB. The HB simulation relies on the use of two AGs (Fig. 7) operating at the same frequency $\omega_{A G 1}=\omega_{A G 2}=\omega_{s}$, with the amplitudes $A_{A G 1}$ and $A_{A G 2}$, respectively. One of these generators is connected to osc $\mathrm{c}_{1}$ and the other one is connected to osc2. Because the two oscillators are in a synchronized regime, there is a constant phase shift $\phi$ between the two. In the AG analysis, the phase of the first AG is arbitrarily set to zero and that of the second AG is taken as a variable, $\phi_{A G 2}=\phi$. Then, the system is analyzed solving the following non-perturbation condition of the two AGs:

$$
\begin{aligned}
& Y_{A G 1}\left(A_{A G 1}, A_{A G 2}, \omega_{s}, \phi\right)=0 \\
& Y_{A G 2}\left(A_{A G 1}, A_{A G 2}, \omega_{s}, \phi\right)=0
\end{aligned}
$$

where $Y_{A G i}$, with $i=1,2$, is the ratio between the current and voltage at each AG. System (23) is an outer-tier condition, which is solved with the pure HB system as an inner tier. Results obtained with (23) have been superimposed in Fig. 8(a) and are nearly overlapped with those obtained with the semi-analytical model (21). The second analysis has been carried out for the antenna gain $G=6 \mathrm{~dB}$. We have used a smaller gain than in the case of self-injection only [Fig. (3)] to evidence that the multi-valued behavior is more pronounced in this case and arises from smaller gain values. The turning points should lead to jumps versus variation in the distance $d_{1}=d_{2}$. Note that, as a rule, turning points arise for the higher gain values and shorter distances, associated with a higher impact of the injection-locking signals. These turning points cannot be directly obtained with the HB simulation, and the use of the AG-based parameter switching [23-24] is cumbersome in this case due to the high number of turning points. The simulation results have been validated experimentally. The measurement set-up is shown in Fig. 6(b). Measurement points have been superimposed in Fig. 8 for the two gain values with an excellent agreement. 


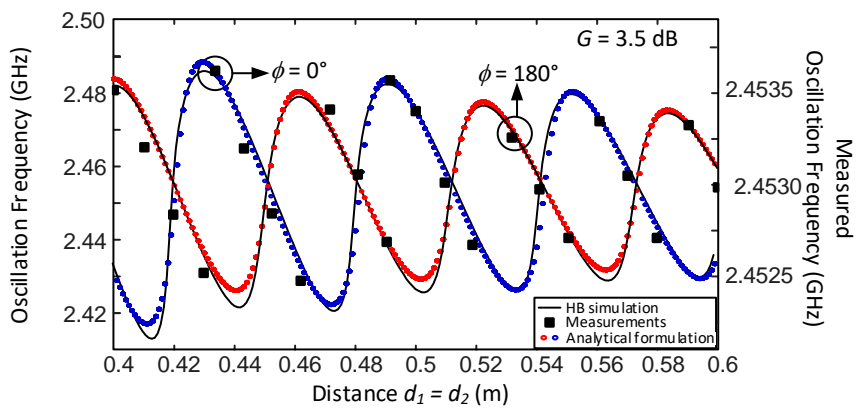

(a)

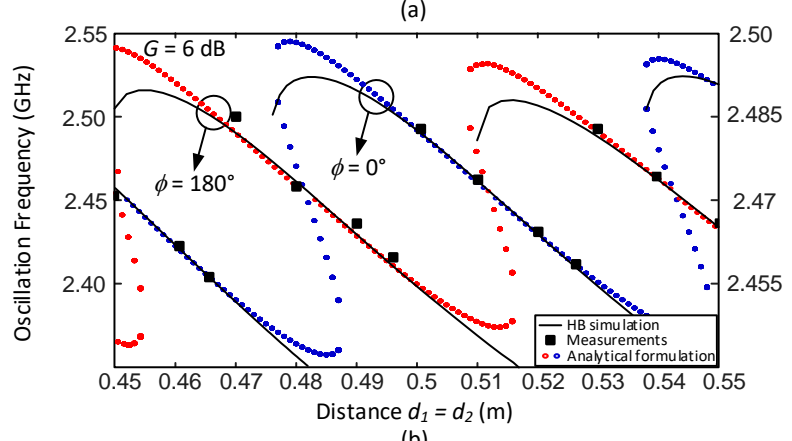

(b)

Fig. 8 Variation of the oscillation frequency solutions with the distance $d$ being $d=d_{1}=d_{2}$ for two different values of antenna gain. (a) $G=3.5 \mathrm{~dB}$ and (b) $G=6 \mathrm{~dB}$. Results obtained with analytical formulation are compared with those provided by HB with 8 harmonic terms. Measurements have been superimposed.

The next analysis considers a more general situation in which only one of the two distances $\left(d_{2}\right)$ is varied, while the other is kept constant at $d_{1}=0.5 \mathrm{~m}$. Even though the oscillators and antenna gains are still assumed equal, the SILOs are no longer in the same conditions, so only the general formulation (19) and (20) can be used. Solutions obtained for the antenna gain $G=6 \mathrm{~dB}$ when varying $d_{2}$ are shown in Fig. 9, where they have been traced in terms of the oscillation phase and oscillation frequency. There are two solutions, in which the respective phase shifts vary about $\phi=0^{\circ}$ and $\phi=180^{\circ}$ when traced versus $d_{2}$, as shown in Fig. 9(b). Circuit-level HB simulations are superimposed. However, due to the presence of turning points, the HB solution curves could not be traced completely.
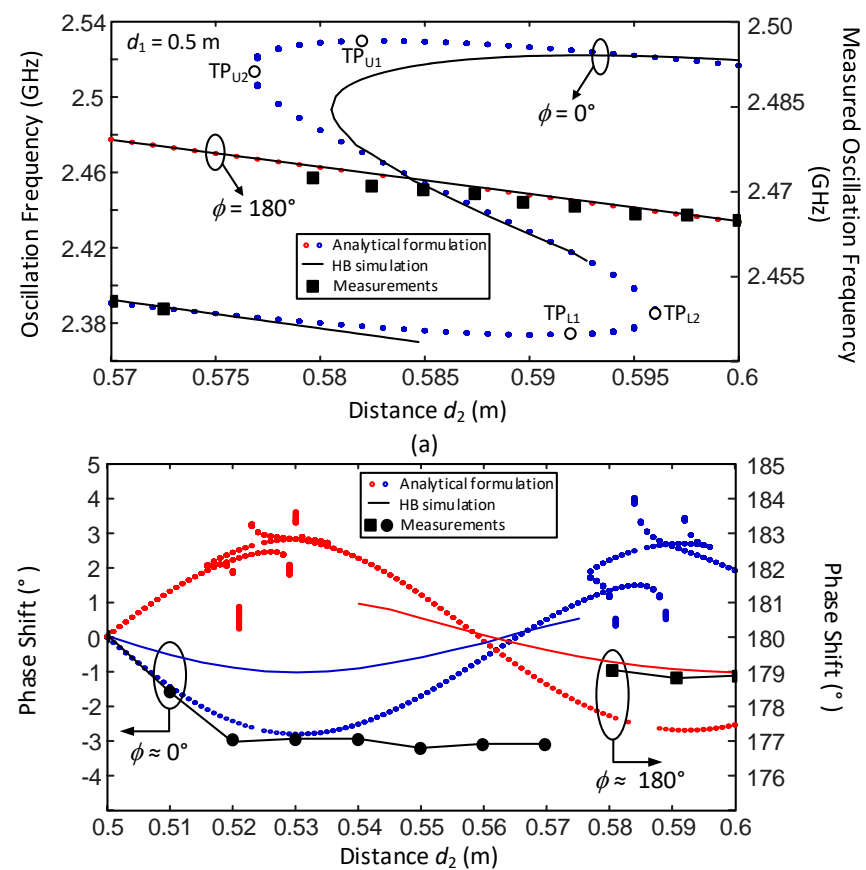

(b)

Fig. 9 Variation of the oscillation frequency solutions with the distance $d_{2}$ for an antenna gain $G=6 \mathrm{~dB}$. . Results obtained with analytical formulation are compared with those provided by HB with 8 harmonic terms. Measurements have been superimposed. (a) Oscillation frequency. (b) Phase shift.

Once the oscillation frequency $\omega_{s}$ and phase shift $\phi$ of each solution are known, the amplitudes at $\operatorname{osc}_{1}$ and $\operatorname{osc}_{2}$, given by $\Delta V_{1}$ and $\Delta V_{2}$, are easily calculated from (18), which depends linearly on these two variables. To compare with the case of an injection-locked oscillator driven by an independent periodic source, the oscillation amplitude has been traced versus the oscillation frequency $\omega_{s}$ in Fig. 10, for the case of identical distances to the target $d_{1}=d_{2}$ and antenna gain $G=3.5 \mathrm{~dB}$. Two different curves are represented: the one corresponding to the phase shift $\phi=0^{\circ}$, in Fig. 10(a), and the one corresponding to $\phi=180^{\circ}$, in Fig. 10(c). Fig. 10(b) and Fig. 10(d) present the results obtained with HB, using two AGs, which show a very good agreement with the semianalytical formulation. For each of the two phase values, the solution curve is spiral-like, but with an ellipsoidal form. It winds around the original free-running oscillator solution (in the absence of any synchronizing signals), which is located about the center of the diagram. This kind of response has some similarity with that of an injection-locked oscillator, which, for moderate input amplitude, exhibits closed solution curves when varying the input frequency [19-20]. In an injection-locked oscillator, each point of the closed curve corresponds to a different phase shift with respect to the input source. In contrast, each point of the wireless synchronization curves in Fig. 10 corresponds to a different distance $d_{1}=d_{2}$.

Due to the coexistence of steady-state solutions derived from the formulation (19) to (21), and confirmed by the circuit-level simulations, the stability analysis will be 
essential. This will allow distinguishing between physical and unphysical sections of the solution curves.

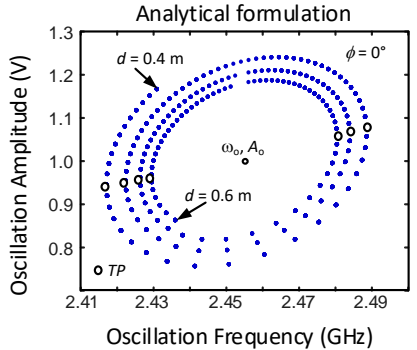

(a)

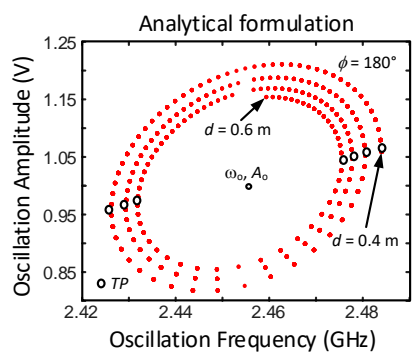

(c)

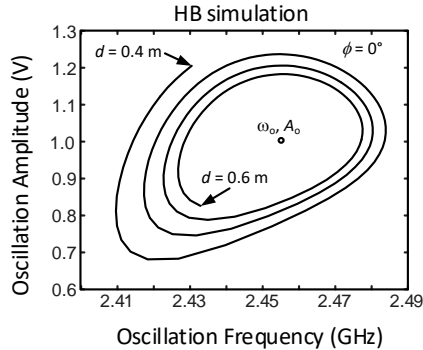

(b)

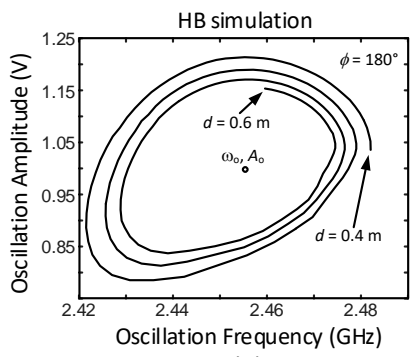

(d)
Fig. 10 Variation of the oscillation amplitudes for the in-phase and $180^{\circ}$ solutions with the distance $d=d_{1}=d_{2}$ and antenna gain $G=3.5 \mathrm{~dB}$. Results obtained with analytical formulation [in (a) and (c)] are compared with those provided by HB with 8 harmonic terms [in (b) and (d)].

\section{STABILITY ANALYSIS}

The stability analysis will be based on the application of a small amplitude perturbation to system (17). The amplitudes and phase values will become $V_{1}+\delta v_{1}(t), V_{2}+\delta v_{2}(t)$, and $\phi_{1}=0+\delta \phi_{1}(t)$ and $\phi_{2}=\phi+\delta \phi_{2}(t)$. To clarify the dependence on the various variables, the steady-state system (17) will be rewritten in terms of the two different phases, $\phi_{1}$ and $\phi_{2}$ :

$$
\begin{aligned}
& Y_{1 v}\left(V_{1}-V_{o 1}\right)+Y_{1 \omega}\left(\omega_{s}-\omega_{o 1}\right)-D_{1} e^{-j \omega_{s} \frac{2 d_{1}}{c}}-C_{1} e^{-j \omega_{s} \frac{d_{T}}{c}} e^{j\left(\phi_{2}-\phi_{1}\right)}=0 \\
& Y_{2 v}\left(V_{2}-V_{o 2}\right)+Y_{2 \omega}\left(\omega_{s}-\omega_{o 2}\right)-D_{2} e^{-j \omega_{s} \frac{2 d_{2}}{c}}-C_{2} e^{-j \omega_{s} \frac{d_{T}}{c}} e^{j\left(\phi_{1}-\phi_{2}\right)}=0
\end{aligned}
$$

where the synchronized-oscillation frequency $\omega_{s}$ is shown explicitly in the exponents. In synchronized-solution curves, instability is usually associated to unlocking phenomena, occurring at turning points, or Hopf bifurcations with a small difference between the fundamental frequency of the original periodic solution $\left(\omega_{s}\right)$ and the newly generated fundamental [19-20, 26-27]. Therefore, assumption of a small frequency increment $s$ is justified, and it will be possible to perform a first-order Taylor of the frequency-dependent functions about the steady-state synchronization frequency $\omega_{s}$. As discussed in Section II, multiplication by the complex frequency $s$ will act like a time differentiator. In oscillator osc $_{1}$, the time differentiator acts on its local signal, through $Y_{\omega}$, leading to the increment $-j \delta \dot{v}_{1}(t) / V_{s 2}+\delta \dot{\phi}_{1}(t)$, on its own signal reflected by the target, through $g_{1 \omega}$, leading to $-j \delta \dot{v}_{1}\left(t-\tau_{1}\right) / V_{s 2}+\delta \dot{\phi}_{1}\left(t-\tau_{1}\right)$ and on the signal transmitted by osc 2 through $\quad C_{1} e^{-j \omega_{s} \frac{d_{T}}{c}}$, leading to $-j \delta \dot{v}_{2}\left(t-\tau_{T}\right) / V_{s 1}+\delta \dot{\phi}_{2}\left(t-\tau_{T}\right)$, where the delays are $\tau_{1}=2 d_{1} / c$ and $\tau_{T}=d_{T} / c$. An identical situation is obtained for the oscillator $\operatorname{osc}_{2}$. Taking this into account, one obtains:

$$
\begin{aligned}
& Y_{1 v} \delta v_{1}(t)+\left(Y_{1 \omega}-g_{1 \omega}\right)\left(-j \frac{\delta \dot{v}_{1}}{V_{s 1}}+y_{1}\right)+ \\
& g_{1 \omega} \tau_{1} \dot{y}_{1}-C_{1 \omega}^{T}\left(-j \frac{\delta \dot{v}_{2}}{V_{s 1}}+y_{2}-\tau_{T} \dot{y}_{2}\right) e^{j\left(\phi_{o}\right)}= \\
& =C_{1} e^{-j \omega_{s} \frac{d_{T}}{c}} j e^{j\left(\phi_{o}\right)}\left(\delta \phi_{2}(t)-\tau_{T} y_{2}-\delta \phi_{1}(t)\right) \\
& Y_{2 v} \delta v_{2}(t)+\left(Y_{2 \omega}-g_{2 \omega}\right)\left(-j \frac{\delta \dot{v}_{2}}{V_{s 2}}+y_{2}\right)+ \\
& g_{2 \omega} \tau_{2} \dot{y}_{2}-C_{2 \omega}^{T}\left(-j \frac{\delta \dot{v}_{1}}{V_{s 2}}+y_{1}-\tau_{T} \dot{y}_{1}\right) e^{j\left(-\phi_{o}\right)}= \\
& =C_{2} e^{-j \omega_{s} \frac{d_{T}}{c}} j e^{-j\left(\phi_{o}\right)}\left(\delta \phi_{1}(t)-\tau_{T} y_{1}-\delta \phi_{2}(t)\right) \\
& \delta \dot{\phi}_{1}=y_{1}, \delta \dot{\phi}_{2}=y_{2}
\end{aligned}
$$

where:

$$
\begin{aligned}
& g_{1 \omega}=\left(\frac{\partial D_{1}}{\partial \omega_{s}}-j D_{1} \frac{2 d_{1}}{c}\right) e^{-\frac{j \omega_{s} 2 d_{1}}{c}} \\
& C_{1 \omega}^{T}=\left(\frac{\partial C_{1}}{\partial \omega_{s}}-j C_{1} \frac{d_{T}}{c}\right) e^{-\frac{j \omega_{s} d_{T}}{c}} \\
& g_{2 \omega}=\left(\frac{\partial D_{2}}{\partial \omega_{s}}-j D_{2} \frac{2 d_{2}}{c}\right) e^{-\frac{j \omega_{s} 2 d_{2}}{c}} \\
& C_{2 \omega}^{T}=\left(\frac{\partial C_{2}}{\partial \omega_{s}}-j C_{2} \frac{d_{T}}{c}\right) e^{-\frac{j \omega_{s} d_{T}}{c}}
\end{aligned}
$$

As in the case of the single SILO, the various time delays, in the order of a few ns, give rise to very small magnitudes when multiplied by the frequency derivatives, so they can be neglected, which leads to the simplified system:

$Y_{1 v} \delta v_{1}(t)+H_{\omega 11}\left(-j \frac{\delta \dot{v}_{1}(t)}{V_{o 1}}+\delta \dot{\phi}_{1}(t)\right)+H_{\omega 12}\left(-j \frac{\delta \dot{v}_{2}(t)}{V_{o 1}}+\delta \dot{\phi}_{2}(t)\right)+$

$F_{1} \delta \phi_{1}(t)-F_{1} \delta \phi_{2}(t)=0$,

$Y_{2 v} \delta v_{2}(t)+H_{\omega 21}\left(-j \frac{\delta \dot{v}_{1}(t)}{V_{o 2}}+\delta \dot{\phi}_{1}(t)\right)+H_{\omega 22}\left(-j \frac{\delta \dot{v}_{2}(t)}{V_{o 2}}+\delta \dot{\phi}_{2}(t)\right)$

$-F_{2} \delta \phi_{1}(t)+F_{2} \delta \phi_{2}(t)=0$

where the following functions have been defined, for compactness:

$$
\begin{aligned}
& H_{\omega 11} \equiv T_{\omega 1}=Y_{1 \omega}-g_{1 \omega}, \quad H_{\omega 12}=-C_{1 \omega}^{T} e^{j \phi_{o}} \\
& F_{1}=j C_{1} e^{-j \omega_{s} \frac{d_{T}}{c}} e^{j \phi_{o}}, H_{\omega 22} \equiv T_{\omega 2}=Y_{2 \omega}-g_{2 \omega} \\
& H_{\omega 21}=-C_{2 \omega}^{T} e^{-j \phi_{o}}, \quad F_{2}=j C_{2} e^{-j \omega_{s} \frac{d_{T}}{c}} e^{-j \phi_{o}}
\end{aligned}
$$


The phase derivatives are calculated at $\phi_{1}=0, \phi_{2}=\phi_{0}$. Splitting (27) into real and imaginary parts and assembling the terms affecting time-differentiated and non-differentiated variables, one obtains the following matrix system in a straight-forward manner:

$$
\left[\begin{array}{c}
\delta \dot{v}_{1} \\
\delta \dot{v}_{2} \\
\delta \dot{\phi}_{1} \\
\delta \dot{\phi}_{2}
\end{array}\right]=[M]\left[\begin{array}{c}
\delta v_{1} \\
\delta v_{2} \\
\delta \phi_{1} \\
\delta \phi_{2}
\end{array}\right]
$$

where $[M]=\left[M_{1}\right]^{-1}\left[M_{2}\right]$ and:

$$
\begin{aligned}
& {\left[M_{1}\right]=\left[\begin{array}{cccc}
H_{\omega 11}^{i} / V_{s 1} & H_{\omega 12}^{i} / V_{s 2} & H_{\omega 11}^{r} & H_{\omega 12}^{r} \\
-H_{\omega 11}^{r} / V_{s 1} & -H_{\omega 12}^{r} / V_{s 2} & H_{\omega 11}^{i} & H_{\omega 12}^{i} \\
H_{\omega 21}^{i} / V_{s 1} & H_{\omega 22}^{i} / V_{s 2} & H_{\omega 21}^{r} & H_{\omega 22}^{r} \\
-H_{\omega 21}^{r} / V_{s 1} & -H_{\omega 22}^{r} / V_{s 2} & H_{\omega 21}^{i} & H_{\omega 22}^{i}
\end{array}\right]} \\
& {\left[M_{2}\right]=\left[\begin{array}{cccc}
-G_{1 v} & 0 & -F_{1}^{r} & F_{1}^{r} \\
-B_{1 v} & 0 & -F_{1}^{i} & F_{1}^{i} \\
0 & -G_{2 v} & F_{2}^{r} & -F_{2}^{r} \\
0 & -B_{2 v} & F_{2}^{i} & -F_{2}^{i}
\end{array}\right]}
\end{aligned}
$$

System (29) is able to predict four poles, corresponding to the four eigenvalues of $[M]$. Due to the free-running behavior of the overall system, one of these poles will necessary be $\lambda_{1}=0$. The rest may be either three real poles or one real pole and a pair of complex-conjugate poles. Because the matrix $[M]$ depends on the coefficients in (26), containing terms with a sinusoidal dependence on $d_{1}, d_{2}$ and $d_{T}$, one may expect a nearly periodical variation of these poles with respect to the distances $d_{1}$ and $d_{2}$.

The above formulation has been applied to the two examples in the previous section, with the steady-state solution curves presented in Fig. 8. Fig. 11 shows the evolution of the real part of the poles versus the distance $d_{1}=d_{2}$ for the antenna gain $G=3.5 \mathrm{~dB}$. Fig. 11(a) corresponds to the solution $\phi=0^{\circ}$ and Fig. 11(b) corresponds to the solution $\phi=180^{\circ}$. In the two cases, there are three real poles, in addition to the pole $\lambda_{1}=0$, due to the system autonomy. Two of these poles are always relatively far from the imaginary axis but the third pole, $\lambda_{2}$, crosses through zero at particular distance values, delimiting the stable and unstable sections of the solution curves. The solutions are unstable for the curve sections where $\lambda_{2}>0$, traced in dashed line in Fig. 8. The pattern has a near-periodicity of about half a wavelength, so broader stable regions would be obtained for a lower value of the carrier frequency $\omega_{s}$.

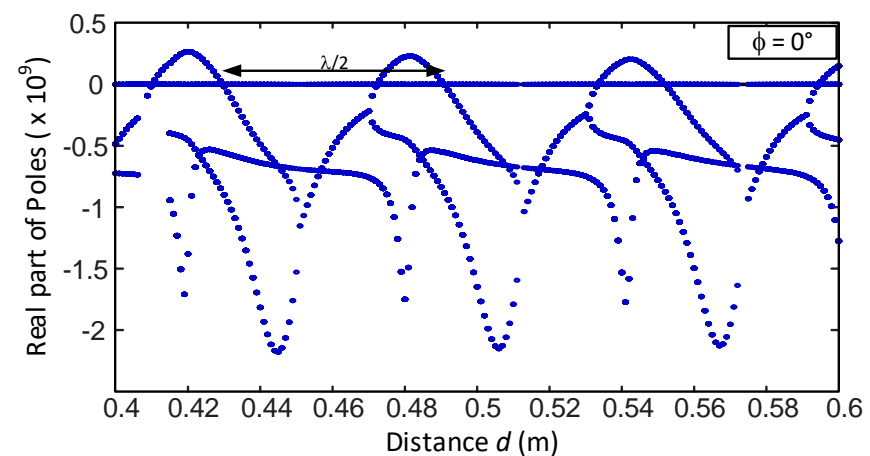

(a)

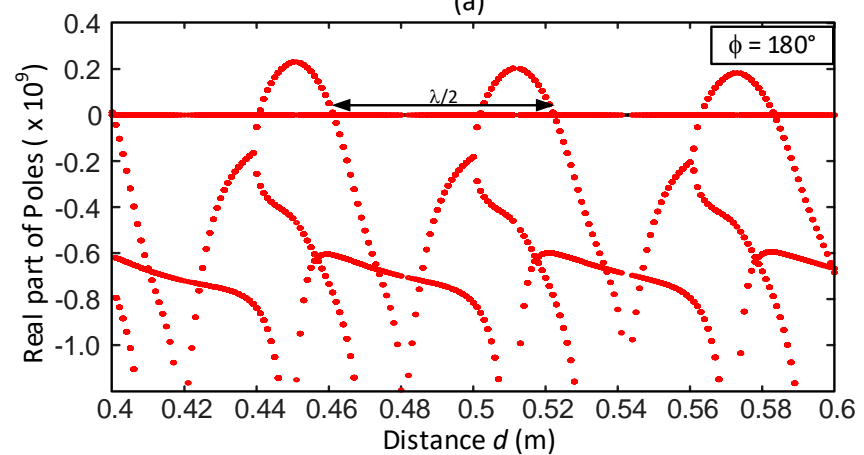

(b)

Fig. 11 Stability analysis of the mutual injection-locked system versus distance $d=d_{1}=d_{2}$ for antenna gain $G=3.5 \mathrm{~dB}$ using analytical formulation. (a) In-phase solution. (b) $180^{\circ}$ solution.

The results obtained with (29) have been rigorously validated with a stability analysis at circuit level, applying pole-zero identification [28-29] to the solutions obtained with HB (Fig. 12). There is consistency between the results obtained with the semi-analytical formulation and with the costly circuit-level analysis. For equal distances to the target, either the solution $\phi=0^{\circ}$ or the solution $\phi=180^{\circ}$ will be stable, depending on the particular distance value.

The crossing of a real pole through zero is usually associated with a turning point of the solution curve versus an analysis parameter. In fact, at points where $\lambda_{2}=0$ the solution curves traced versus the oscillation frequency $\omega_{s}$ in Fig. 10 exhibit an infinite slope. For the $d=d_{1}=d_{2}$ interval considered, there are seven turning points, in full agreement with the seven crossings through zero of the real pole $\lambda_{2}$ in Fig. 11. The upper sections of the spiral-like curves [in Fig. 10(a) and Fig. 10(c)] are stable, whereas the lower sections are unstable, agreeing with the most common situation in injection-locked oscillators [30]. The results of the stability analysis have also been validated experimentally. Fig. 13 shows the two individual oscillator waveforms obtained for the respective distances to the target $d_{1}=d_{2}=0.45 \mathrm{~m}$ and $d_{1}=d_{2}=0.48 \mathrm{~m}$. When varying the distance, the experimental solution undergoes a $180^{\circ}$ phase shift in agreement with the predictions in Fig. 11 and Fig. 12. 


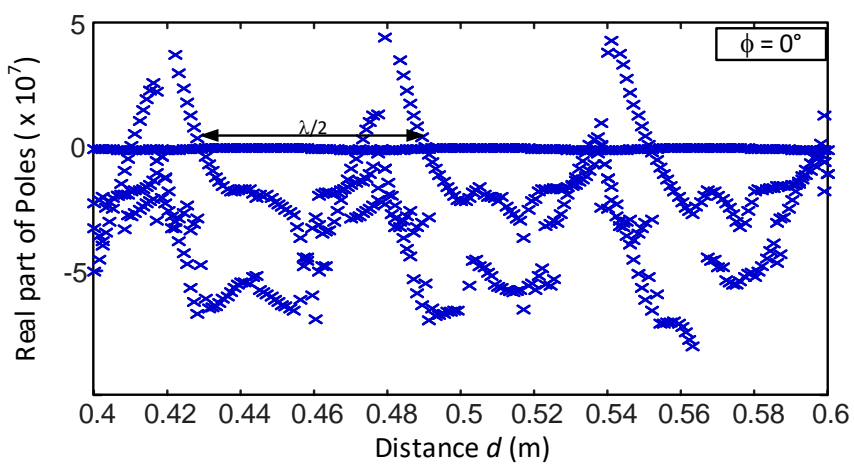

(a)

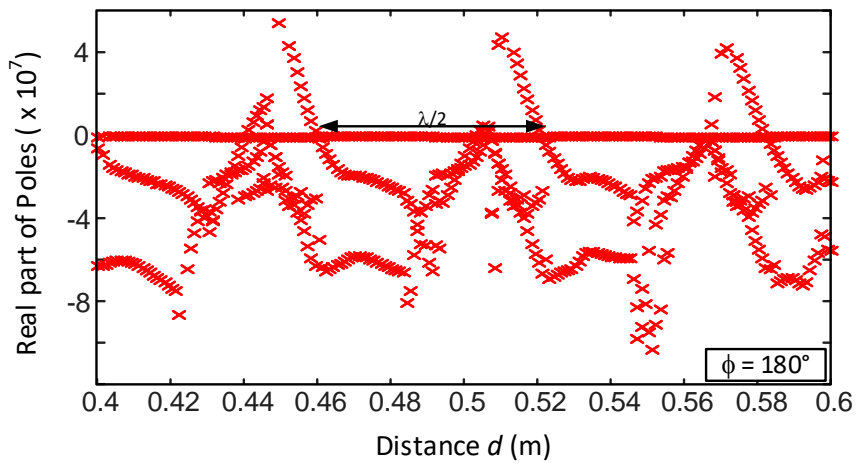

(b)

Fig. 12 Stability analysis at circuit level of the mutually injection-locked system versus distance $d=d_{1}=d_{2}$ for antenna gain $G=3.5 \mathrm{~dB}$, using polezero identification [28-29]. (a) In-phase solution. (b) $180^{\circ}$ solution.

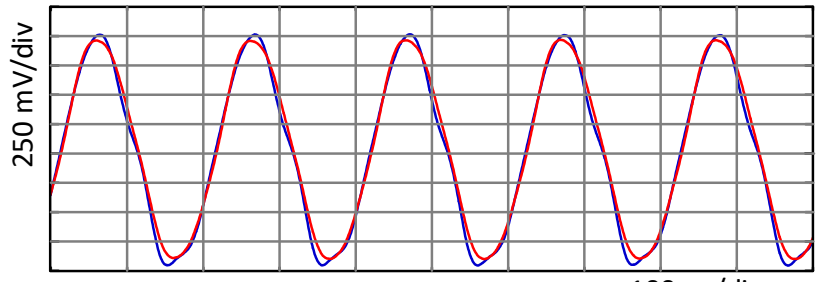

(a)

$100 \mu \mathrm{s} / \mathrm{div}$

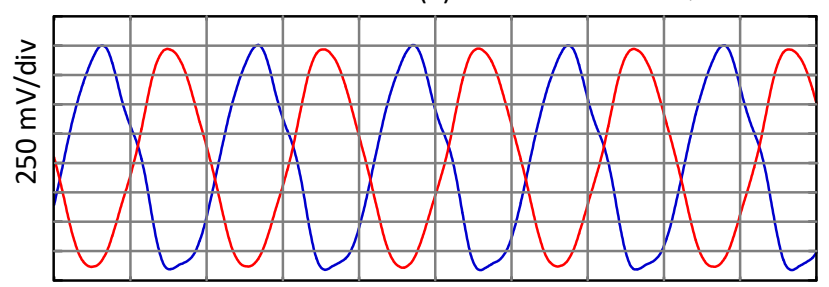

(b)

Fig. 13 Experimental measurement of the individual oscillator waveforms. (a) $d_{1}=d_{2}=0.45 \mathrm{~m}$, in phase. (b) $d_{1}=d_{2}=0.48 \mathrm{~m}$, with $180^{\circ}$ phase shift.

Next, the case of different distances $d_{1} \neq d_{2}$, corresponding to the solution curves in Fig. 9, obtained for $G=6 \mathrm{~dB}$, has been considered. In this case, the curves exhibit a turning point versus $d_{2}$, in addition to the turning point versus $\omega_{s}$. In consistency with this, two real poles cross the imaginary axis through zero, as shown in Fig 14(a), where the real part of the four poles has been traced versus $d_{2}$. As in the previous case, results obtained with (29) have been rigorously validated with pole-zero identification [28-29], at circuit level [Fig. 14(b)]. When varying $d_{2}$, while keeping $d_{1}$ constant at $d_{1}=0.5 \mathrm{~m}$,

the system undergoes desynchronization at particular $d_{2}$ values, which leads to a quasi-periodic solution. The phenomenon is associated to desynchronization occurring at turning points of the solution curves, as in the case of ordinary injection-locked oscillators excited with small-amplitude (independent) synchronizing signals [31]. This phenomenon has been validated experimentally. Fig. 15 shows the spectra measured for two different values of $d_{2}$. For $d_{2}=0.57 \mathrm{~m}$ the system exhibits the expected synchronized behavior. For $d_{2}=0.576 \mathrm{~m}$, the spectrum shows the typical triangular shape associated with the desynchronization.

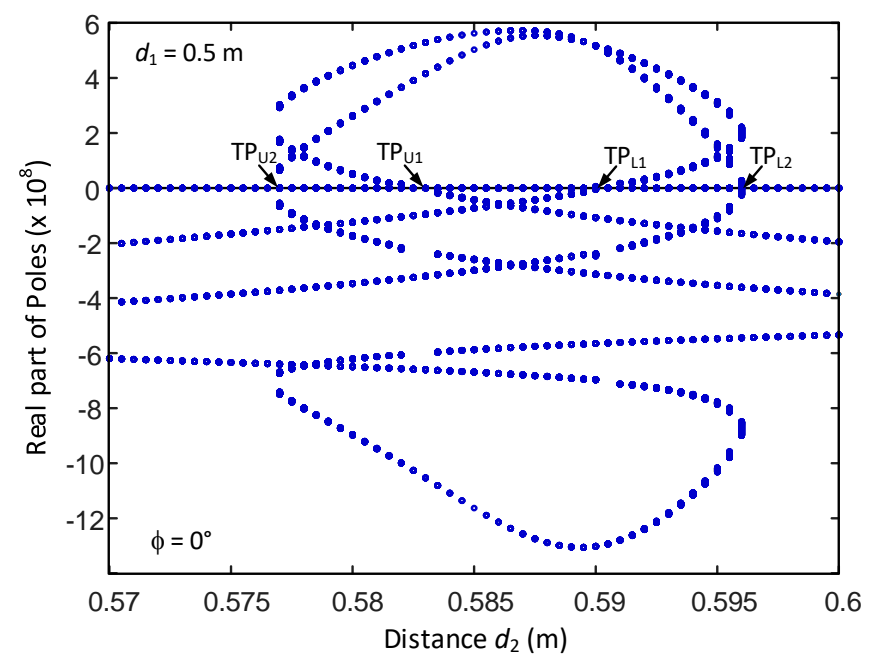

(a)

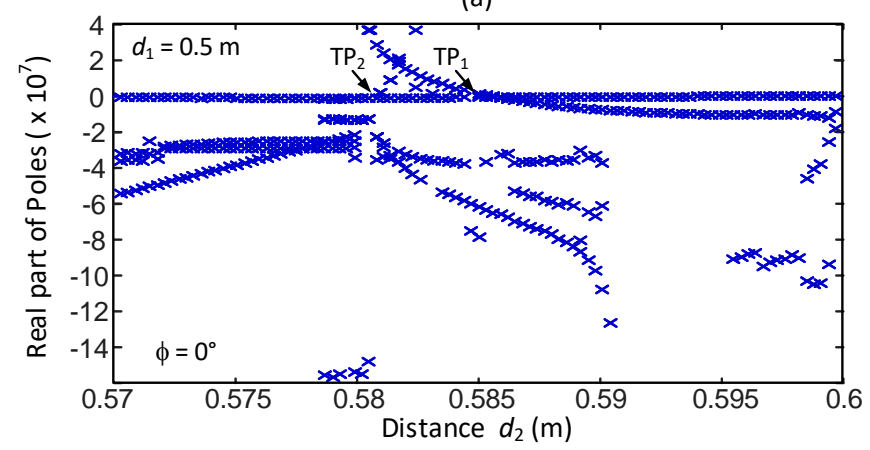

(b)

Fig. 14 Stability analysis of the solution curve $\phi=0^{\circ}$ in Fig. 9, for an antenna gain $G=6 \mathrm{~dB}$ and different distances. (a) Results using the analytical formulation in (27). (b) Results obtained with circuit-level simulations based on pole-zero identification [28-29]. 


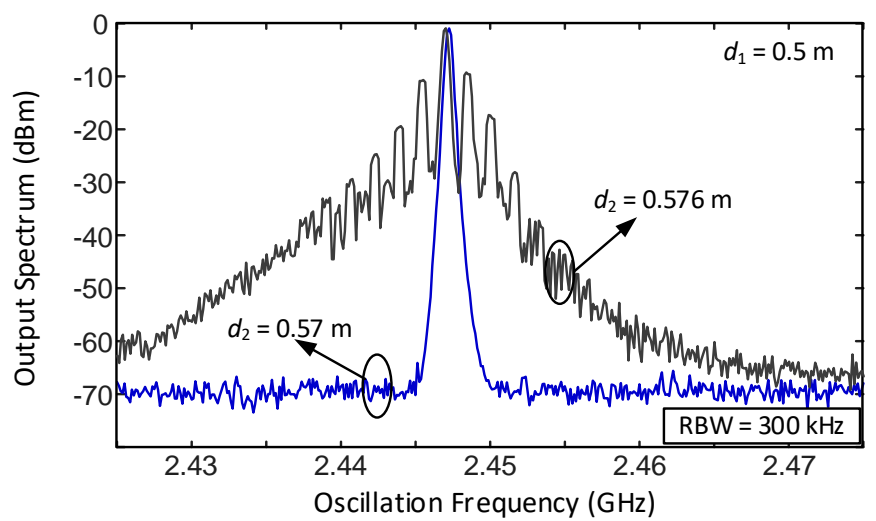

Fig. 15 Spectra measured for $G=6 \mathrm{~dB}$, with different distances to the target, $d_{1}$ and $d_{2}$. The resolution bandwidth of the measured spectra is $\mathrm{RBW}=300 \mathrm{kHz}$.

\section{Phase Noise Analysis}

The phase-noise analysis will be based on the perturbation of system (17) in the presence of noise sources. Only white noise sources will be initially considered to get some insight into the dependence on the antenna gain and the distances to the target and between the oscillator elements. The noise contributions of each oscillator will be modeled with an equivalent current source connected in parallel at the analysis node [25]. There will be two different white noise current sources, given by $I_{w 1}$ and $I_{w 2}$. The perturbed system is:

$$
\left[M_{1}\right]\left[\begin{array}{c}
\delta \dot{v}_{1} \\
\delta \dot{v}_{2} \\
\delta \dot{\phi}_{1} \\
\delta \dot{\phi}_{2}
\end{array}\right]-\left[M_{2}\right]\left[\begin{array}{c}
\delta v_{1} \\
\delta v_{2} \\
\delta \phi_{1} \\
\delta \phi_{2}
\end{array}\right]=\left[\begin{array}{c}
\frac{I_{w 1}^{r}(t)}{V_{s 1}} \\
\frac{I_{w 1}^{i}(t)}{V_{s 1}} \\
\frac{I_{w 2}^{r}(t)}{V_{s 2}} \\
\frac{I_{w 2}^{i}(t)}{V_{s 2}}
\end{array}\right]
$$

where the phase derivatives are evaluated at the steady-state values $\phi_{1}=0, \phi_{2}=\phi_{o}$ and the matrixes $\left[M_{1}\right]$ and $\left[M_{2}\right]$ were defined in (30). The phase-noise spectrum is obtained through application of the Fourier transform to the above system, taking into account that the two noise sources are uncorrelated and so are the real and imaginary parts of each of these two noise sources [21]. This provides the following spectra for the amplitude and phase noise of each oscillator:

$$
\begin{aligned}
& {\left[\left\langle\left|\delta v_{1}(\Omega)\right|^{2}\right\rangle\left\langle\left|\delta v_{2}(\Omega)\right|^{2}\right\rangle\left\langle\left|\delta \phi_{1}(\Omega)\right|^{2}\right\rangle\left\langle\left|\delta \phi_{2}(\Omega)\right|^{2}\right\rangle\right]^{\mathrm{T}}=} \\
& \operatorname{diag}\left\{\left[M_{n}(j \Omega)\right]^{-1}\left\langle\bar{N}(\Omega) \bar{N}^{+}(\Omega)\right\rangle\left\{\left[M_{n}(j \Omega)\right]^{-1}\right\}^{+}\right\}
\end{aligned}
$$

where the conversion matrix is given by: $\left[M_{n}(j \Omega)\right]=j \Omega\left[M_{1}\right]-\left[M_{2}\right]$ and $\bar{N}$ is the vector containing the equivalent noise sources in (31). To get some insight into the effect of the mutual synchronization, admittance functions of the form $Y(V, \omega)=Y^{r}(V)+j Y^{i}(\omega)$ will be assumed, that is, with the real (imaginary) part depending only on the oscillation amplitude (frequency). Neglecting the amplitude variations, the simplified perturbed system is:

$$
\left\{\left[\begin{array}{cc}
H_{\omega 11}^{i} j \Omega & H_{\omega 12}^{i} j \Omega \\
H_{\omega 21}^{i} j \Omega & H_{\omega 22}^{i} j \Omega
\end{array}\right]+\left[\begin{array}{cc}
-F_{1}^{i} & F_{1}^{i} \\
F_{2}^{i} & -F_{2}^{i}
\end{array}\right]\right\}\left[\begin{array}{c}
\Delta \phi_{1} \\
\Delta \phi_{2}
\end{array}\right]=\left[\begin{array}{c}
I_{w 1}^{i} \\
V_{s 1} \\
I_{w 2}^{i} \\
V_{s 2}
\end{array}\right]
$$

In the case of two identical oscillator and antennas, and under the assumption of $d_{1}=d_{2}$, one obtains the following simplified expression:

$$
\begin{aligned}
& \left\langle\left|\delta \phi_{1}\right|^{2}\right\rangle= \\
& \frac{N\left(\left[T_{\omega 1}^{i}{ }^{2}+\left(C_{1 \omega}^{T, i}\right)^{2}\right] \Omega^{2}+2 C_{1}^{2} \cos ^{2}\left(\omega_{s} \frac{d_{T}}{c}\right)\right)}{\left[T_{\omega 1}^{i 2}+\left(C_{1 \omega}^{T, i}\right)^{2}\right]^{2} \Omega^{4}+4 \Omega^{2} C_{1}^{2}\left(T_{\omega 1}^{i}+C_{1 \omega}^{T, i}\right)^{2} \cos ^{2}\left(\omega_{s} \frac{d_{T}}{c}\right)}
\end{aligned}
$$

where the white-noise spectral density of the two oscillators is assumed equal and $N=2\left|I_{w}\right|^{2} / V_{s}^{2}$. In the absence of a mutual injection locking, the above expression particularizes to:

$$
\left\langle|\delta \phi(\Omega)|^{2}\right\rangle=\frac{N}{T_{\omega 1}^{i 2} \Omega^{2}}
$$

where the assumptions of an admittance function of the form $Y(V, \omega)=Y^{r}(V)+j Y^{i}(\omega)$ and negligible amplitude perturbations have been maintained. As gathered from (34), the mutual synchronization will give rise to a phase-noise reduction with respect to that obtained in a single SILO, due to the additional coefficients in the denominator, associated with the interaction between the two oscillators. The phase noise will also exhibit a quasi-sinusoidal variation with the total distance $d_{T}$.

The analysis of the practical oscillator system has been carried considering flicker noise, with the aid of an additional baseband equation, as in the case of the single SILO. The results for antenna gain $G=6 \mathrm{~dB}$ and equal distances $d_{1}=d_{2}=0.5 \mathrm{~m}$ are shown in Fig. 16, where the phase-noise spectrum can be compared with the one obtained with a single SILO. As expected, the phase noise is lower in the case of two mutually coupled SILOs, with a reduction of about $6 \mathrm{~dB}$. The experimental spectra obtained in the two cases are superimposed with good agreement. 


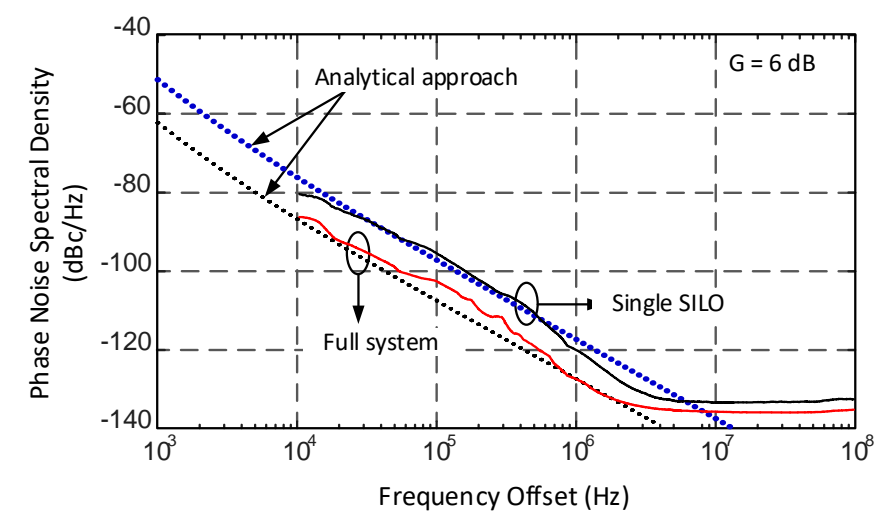

Fig. 16 Phase-noise analysis for antenna gain $G=6 \mathrm{~dB}$ and equal distances $d_{1}=d_{2}=0.5 \mathrm{~m}$. The phase-noise spectra of two mutually locked SILO are compared with the one obtained with a single SILO. The experimental spectra are superimposed.

\section{ENVELOPE-TRANSIENT ANALYSIS}

The system behavior under the instantaneous target movement will be investigated with an envelope-transient analysis, relying on the formulation in (24). The frequencydependent coefficients $H(\omega)$ (where $H$ stands here for any of the frequency dependent functions), affecting the modulated signals, will give rise [32] to the terms $H(\omega) \tilde{V}(t)-j H_{\omega}(\omega) \dot{\tilde{V}}(t)$, where $\tilde{V}(t)$ is the envelope of the modulated signal.

In [12-13], two different types of movement are considered: the back and forth movement, $\Delta d(t)$, and the inward and outward movement, $x(t)$. In the presence of these two movements, the instantaneous distances from each of the two oscillators to the target are different, and given by:

$$
\begin{aligned}
& d_{1}{ }^{\prime}=d_{1}-\Delta d(t)-x(t)=d_{1}+l_{1}(t) \\
& d_{2}{ }^{\prime}=d_{2}+\Delta d(t)-x(t)=d_{2}+l_{2}(t)
\end{aligned}
$$

On the other hand, the distance between the two oscillators remains unchanged at $d_{T}$. The system is formulated:

$$
\begin{aligned}
& Y_{1 v} \Delta V_{1}(t)+\left(Y_{1 \omega}-g_{1 \omega}\right)\left(-j \frac{\Delta \dot{V}_{1}}{V_{o 1}}+\dot{\phi}_{1}\right)+ \\
& g_{1 \omega} \tau_{d 1} \ddot{\phi}_{1}-C_{1 \omega}^{T}\left(-j \frac{\Delta \dot{V}_{2}}{V_{o 1}}+\dot{\phi}_{2}-\tau_{d T} \ddot{\phi}_{2}\right) e^{j \phi_{o}}= \\
& =D_{1} e^{-j \omega_{s}\left(\frac{2 d_{1}}{c}+\frac{2 l_{1}(t)}{c}\right)}-D_{1} e^{-j \omega_{s}\left(\frac{2 d_{1}}{c}\right)}+ \\
& g_{1 \omega}\left(-j \omega_{s} \frac{2 \dot{l}_{1}(t)}{c}\right)+C_{1} e^{-j \omega_{s} \frac{d_{T}}{c}} e^{j\left(\phi_{2}(t)-\tau_{d T} \dot{\phi}_{2}(t)-\phi_{1}(t)\right)} \\
& Y_{2 v} \Delta V_{2}(t)+\left(Y_{2 \omega}-g_{2 \omega}\right)\left(-j \frac{\Delta \dot{V}_{2}}{V_{o 2}}+\dot{\phi}_{1}\right)+ \\
& g_{2 \omega} \tau_{d 2} \ddot{\phi}_{2}-C_{2 \omega}^{T}\left(-j \frac{\Delta \dot{V}_{1}}{V_{o 2}}+\dot{\phi}_{1}-\tau_{d T} \ddot{\phi}_{1}\right) e^{-j \phi_{o}}= \\
& =D_{2} e^{-j \omega_{s}\left(\frac{2 d_{2}}{c}+\frac{2 l_{2}(t)}{c}\right)}-D_{2} e^{-j \omega_{s}\left(\frac{2 d_{2}}{c}\right)}+ \\
& g_{2 \omega}\left(-j \omega_{s} \frac{2 l_{2}(t)}{c}\right)+C_{2} e^{-j \omega_{s} \frac{d_{T}}{c}} e^{j\left(\phi_{1}(t)-\tau_{d T} \dot{\phi}_{1}(t)-\phi_{2}(t)\right)}
\end{aligned}
$$

where the effect of all the time delays on the modulation envelopes has been considered for completeness. However, as already reasoned, this effect can be neglected for low modulation frequencies, which provides the simplified system:

$$
\begin{aligned}
& Y_{1 v} \Delta V_{1}(t)+\left(Y_{1 \omega}-g_{1 \omega}\right)\left(-j \frac{\Delta \dot{V}_{1}}{V_{o 1}}+\dot{\phi}_{1}\right)+ \\
& -C_{1 \omega}^{T}\left(-j \frac{\Delta \dot{V}_{2}}{V_{o 1}}+\dot{\phi}_{2}\right) e^{j \phi_{o}}= \\
& =D_{1} e^{-j \omega_{s}\left(\frac{2 d_{1}}{c}+\frac{2 l_{1}(t)}{c}\right)}-D_{1} e^{-j \omega_{s}\left(\frac{2 d_{1}}{c}\right)}+C_{1} e^{-j \omega_{s} \frac{d_{T}}{c}} e^{j\left(\phi_{2}(t)-\phi_{1}(t)\right)} \\
& Y_{2 v} \Delta V_{2}(t)+\left(Y_{2 \omega}-g_{2 \omega}\right)\left(-j \frac{\Delta \dot{V}_{2}}{V_{o 2}}+\dot{\phi}_{1}\right)+ \\
& -C_{2 \omega}^{T}\left(-j \frac{\Delta \dot{V}_{1}}{V_{o 2}}+\dot{\phi}_{1}\right) e^{-j \phi_{o}}= \\
& =D_{2} e^{-j \omega_{s}\left(\frac{2 d_{2}}{c}+\frac{2 l_{2}(t)}{c}\right)}-D_{2} e^{-j \omega_{s}\left(\frac{2 d_{2}}{c}\right)}+ \\
& C_{2} e^{-j \omega_{s} \frac{d_{T}}{c}} e^{j\left(\phi_{1}(t)-\tau_{d T} \dot{\phi}_{1}(t)-\phi_{2}(t)\right)}
\end{aligned}
$$

where the effect of the velocities $i_{1}(t)$ and $i_{2}(t)$ has also been neglected. The accuracy of the reduced-order system (38) has been verified through comparison with the result of the envelope-transient method at circuit level [32]. A highmodulation frequency has been used for this comparison to enable circuit-level simulations with a reasonable computational cost. This is because the time step must be sufficiently short to ensure a good convergence of the nonlinear envelope-domain system, which would give rise to a huge computational burden for a low modulation frequency. In addition, the instantaneous variation of the oscillation frequency contributes to the envelope modulation. 
Fig. 17 shows a comparison of the phase variation at the oscillator element osc 1 provided by the semi-analytical model (38) and the costly circuit-level simulation in commercial HB. The modulation frequency considered is $f_{m}=1 \mathrm{MHz}$ in all cases. We have chosen $1 \mathrm{MHz}$ just to be able to compare the accuracy of our semi-analytical approach with the results obtained with a circuit-level envelope-transient simulation on commercial HB. This comparison would be virtually impossible at the low modulation frequencies existing in usual motion-sensing applications. This is due the small time step required for the system integration, in comparison with the time rate of the target motion. Fig. 17(a) and Fig. 17(c) show the phase variation under both self-injection and mutual injection effects for maximum displacement $\Delta d=40 \mathrm{~mm}$ and $\Delta d=20 \mathrm{~mm}$, respectively. Fig. 17(b) and Fig. 17(d) show the phase variation under self-injection effects only for maximum displacement $\Delta d=40 \mathrm{~mm}$ and $\Delta d=20 \mathrm{~mm}$. In all cases, the results obtained with the circuit-level simulation and the reduced-order model are nearly overlapped. Therefore, the new formulation should constitute a useful tool for the investigation and optimization of these novel motion-sensing systems.

Finally, Fig. 18 shows the voltage signals obtained when considering a low frequency modulation $f_{m}=10 \mathrm{~Hz}$ and connecting a delay-line demodulator to the output of one of the oscillator circuits. Results are compared with those obtained experimentally.

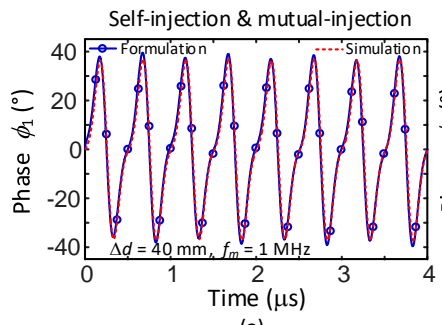

(a)

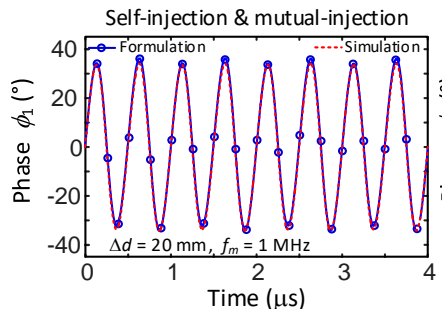

(c)

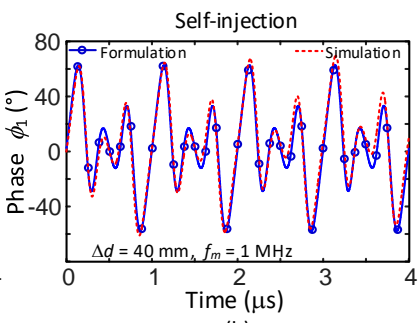

(b)

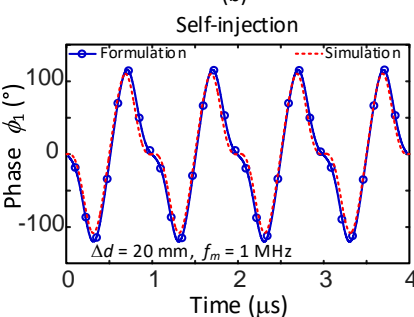

(d)
Fig. 17 Comparison of the envelope-transient results for antenna gain $\mathrm{G}=3.5 \mathrm{~dB}$. Back and forth movement considering self-injection and mutualinjection effects, in (a) and (c), and considering self-injection effects only, in (b) and (d).

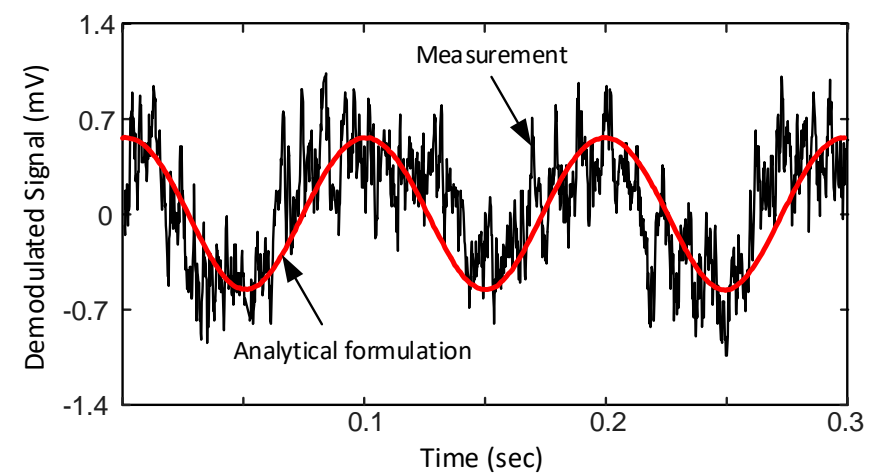

Fig. 18 Voltage signals obtained when considering a low frequency modulation $f_{m}=10 \mathrm{~Hz}$ and connecting a delay-line demodulator to the output of one of the oscillator circuits. Measurement results are superimposed.

\section{CONCLUSION}

A new formulation has been presented for the efficient analysis of self-injection and mutually locked oscillator circuits. It provides insight into the variation of the steadystate solutions versus crucial parameters such as the distance and antenna gain. The formulation evidences the existence of sinusoidal dependences on the distances to the target and the distance between the oscillator elements, which lead to the observation of turning points under a sufficient antenna gain. It also shows the existence of two possible values of phase shift between the oscillator elements, which is associated with the synchronized operation. The formulation has been extended to cover essential aspects of the system behavior, such as stability and phase noise. All the results have been thoroughly validated with computationally expensive circuitlevel simulations and measurements. An envelope-domain formulation has also been presented to analyze the phase modulation due to the target movement. It has been thoroughly compared with envelope-transient simulations based on harmonic balance, applicable only for a high modulation frequency.

\section{REFERENCES}

[1] J. C. Lin, "Noninvasive microwave measurement of respiration," Proc. IEEE, vol. 63, no. 10, pp. 1530-1530, Oct. 1975.

[2] A. D. Droitcour, O. Boric-Lubecke, V. M. Lubecke, J. Lin, and G. T. A. Kovac, "Range correlation and I/Q performance benefits in single-chip silicon doppler radars for non-contact cardiopulmonary monitoring," IEEE Trans. Microw. Theory Techn., vol. 52, no. 3, pp. 838-848, Mar. 2004.

[3] A. Droitcour, Non-contact measurement of heart and respiration rates with a single-chip microwave doppler radar, Ph.D. Thesis, Stanford University, June 2006.

[4] C. Li and J. Lin, "Random body movement cancellation in Doppler radar vital sign detection," IEEE Trans. Microw. Theory Techn., vol.56, no.12, pp.3143-3152, Dec. 2008.

[5] B.J. Jang, S.H. Wi, J.G. Yook, M.Q. Lee, and K.J. Lee, "Wireless bio-radar sensor for heartbeat and respiration detection," Prog. Electromagn. Res., vol. 5, 149-168, 2008.

[6] A. D. Droitcour, O. Boric-Lubecke and G.T.A. Kovacs, "Signalto-Noise Ratio in Doppler Radar System for Heart and Respiratory Rate Measurements," IEEE Trans. Microw. Theory Techn., vol.57, no.10, pp.2498-2507, Oct. 2009.

[7] T. Y. J. Kao, Y. Yan, T. M. Shen, A. Y. K. Chen and J. Lin, "Design and Analysis of a 60-GHz CMOS Doppler Micro-Radar 
System-in-Package for Vital-Sign and Vibration Detection," IEEE Trans. Microw. Theory Techn., vol. 61, no. 4, pp. 16491659, April 2013.

[8] F.K. Wang, C.J. Li, C.H. Hsiao, T.S. Horng, J. Lin, K.C. Peng, J.K. Jau, J.Y. Li and C.C. Chen, "A Novel Vital-Sign Sensor Based on a Self-Injection-Locked Oscillator,” IEEE Trans. Microw. Theory Techn., vol.58, no.12, pp. 4112-4120, Dec. 2010.

[9] F. K. Wang, C. H. Fang, T. S. Horng, K. C. Peng, J. Y. Li and C. C. Chen, "Concurrent Vital Sign and Position Sensing of Multiple Individuals Using Self-Injection-Locked Tags and Injection-Locked I/Q Receivers With Arctangent Demodulation," IEEE Trans. Microw. Theory Techn., vol. 61, no. 12, pp. 4689-4699, Dec. 2013.

[10] F. K. Wang, T. S. Horng, K. C. Peng, J. K. Jau, J. Y. Li and C. C. Chen, "Detection of Concealed Individuals Based on Their Vital Signs by Using a See-Through-Wall Imaging System With a Self-Injection-Locked Radar," IEEE Trans. Microw. Theory Techn., vol. 61, no. 1, pp. 696-704, Jan. 2013.

[11] C. Li, V. M. Lubecke, O. Boric-Lubecke and J. Lin, "A Review on Recent Advances in Doppler Radar Sensors for Noncontact Healthcare Monitoring," IEEE Trans. Microw. Theory Techn., vol. 61, no. 5, pp. 2046-2060, May 2013.

[12] F.K. Wang, T.S. Horng, K.C. Peng, J.K. Jau, J.Y Li and C.C Chen, "Single-Antenna Doppler Radars Using Self and Mutual Injection Locking for Vital Sign Detection with Random Body Movement Cancellation," IEEE Trans. Microw. Theory Techn., vol.59, no.12, pp.3577-3587, Dec. 2011.

[13] F.K. Wang, T.S. Horng, K.C. Peng J.k. Jau, J.Y. Li and C.C. Chen, "Mutual Injection-locked SIL Sensor Array for Vital Sign Detection with random Body Movement Cancellation," IEEE MTT-S Int. Microwave Symp., Baltimore, MD, Jun. 2011.

[14] R. Adler, “A study of locking phenomena in oscillators," Proc. IRE, vol.34, no.6, pp.351-357, Jun.1946.

[15] Heng-Chia Chang, "Stability analysis of self-injection-locked oscillators," IEEE Trans. Microw. Theory Techn., vol. 51, no. 9, pp. 1989-1993, Sept. 2003.

[16] J. L. Altman, "A technique for stabilizing microwave oscillators,” IEEE Trans. on Microw. Theory Tech., vol. MTT-2, no. 2, pp. 16-25, Jul. 1954.

[17] M. Pontón and A. Suárez, "Analysis of self-injection locked oscillators for motion sensing applications," IEEE MTT-S Int. Microwave Symp., San Francisco, CA, May 2016.

[18] F. Ramírez, E. de Cos and A. Suárez, "Nonlinear analysis tools for the optimized design of harmonic-injection frequency dividers”, IEEE Trans. Microw. Theory Techn., vol. 51, no. 6, pp. 1752-1762, Jun. 2003.

[19] A. Suárez, Analysis and Design of Autonomous Microwave Circuits. Hoboken, NJ: Wiley IEEE Pres, 2009.

[20] A. Suárez, R. Quéré, Stability Analysis of Nonlinear Microwave Circuits, Artech-House Publishers, Boston, 2003.

[21] C. Balanis, Antenna Theory. Analysis and Design. John Wiley \& Sons, 2005.

[22] K. Kurokawa, "Some basic characteristics of broadband negative resistance oscillators," Bell Syst. Tech. J., vol. 48, pp. 19371955, Jul.-Aug., 1969.

[23] A. Suárez and F. Ramírez, "Analysis of stabilization circuits for phase-noise reduction in microwave oscillators," IEEE Trans. Microw. Theory Techn., vol.53, no.9, pp.2743-2751, Sept. 2005.

[24] F. Ramírez, M. Pontón, S. Sancho and A. Suárez, "Phase-Noise Analysis of Injection-Locked Oscillators and Analog Frequency Dividers”, IEEE Trans. Microw. Theory Techn., vol. 56, no.2, pp. 393-407, Feb., 2008.

[25] A. Suárez, F. Ramírez and S. Sancho, "Stability and Noise Analysis of Coupled-Oscillator Systems," IEEE Trans. Microw. Theory Techn., vol. 59, no. 4, pp. 1032-1046, April 2011.
[26] J. Guckenheimer and P. Holmes, Nonlinear Oscillations, Dynamic Systems, and Bifurcations of Vector Fields. New York: Springer-Verlag, 1983.

[27] G. Ioos, D. Joseph, Elementary Stability and Bifurcation Theory. New York. Springer, 1980.

[28] J. Jugo, J. Portilla, A. Anakabe, A. Suárez, and J. M. Collantes, "Closed-loop stability analysis of microwave amplifiers," Electron. Lett., vol. 37, no. 4, pp. 226-228, Mar. 2001.

[29] J. M. Collantes, I. Lizarraga, A. Anakabe, and J. Jugo, "Stability verification of microwave circuits through Floquet multiplier analysis”, Proc. IEEE Asia-Pacific Circuits Syst., 2004, pp. 997-1000.

[30] R. Quéré, E. Ngoya, M. Camiade, A. Suarez, M. Hessane and J. Obregon, "Large signal design of broadband monolithic microwave frequency dividers and phase-locked oscillators," IEEE Trans. Microwave Theory Techn., vol. 41, no. 11, pp. 1928-1938, Nov., 1993.

[31] A. Suarez, J. Morales and R. Quéré, "Synchronization analysis of autonomous microwave circuits using new global-stability analysis tools," IEEE Trans. on Microwave Theory and Techn., vol. 46, no. 5, pp. 494-504, May 1998.

[32] E. Ngoya and R. Larcheveque, "Envelope transient analysis: A new method for the transient and steady-state analysis of microwave communication circuits and systems," IEEE MTT-S Int. Microwave Symp., San Francisco, CA, 1996, pp. 1365-1368.

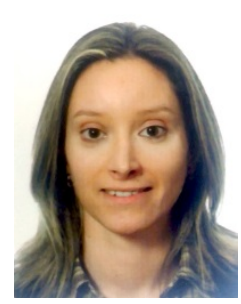

Mabel Pontón (S’08-M’11) was born in Santander, Spain. She received the Telecommunication Engineering degree from the University of Cantabria, Spain in 2004, Masters in Information Technologies and Wireless Communications Systems and Ph.D. degree from the University of Cantabria, Santander, Spain, in 2008 and 2010, respectively. In 2006, she joined the Communications Engineering Department of University of Cantabria.

From 2011 to 2013 she was with the Group of Electronic Design and Applications (EDA) at Georgia Institute of Technology, Atlanta, GA as a Post-Doctoral Research Fellow. Her research interests are focused on the nonlinear analysis and simulation of radiofrequency and microwave circuits, with emphasis on phase-noise, stability and bifurcation analysis of complex oscillator topologies.

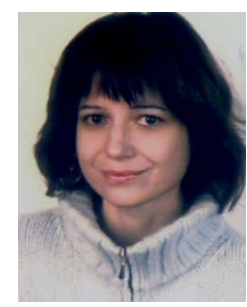

Almudena Suárez (M'96-SM’01-F'12) was born in Santander, Spain. She received the Electronic Physics and Ph.D. degrees from the University of Cantabria, Santander, Spain, in 1987 and 1992, respectively, and the Ph.D. degree in electronics from the University of Limoges, Limoges, France, in 1993.

She is currently a Full Professor with the Communications Engineering Department, University of Cantabria. She co-authored Stability Analysis of Nonlinear Microwave Circuits (Artech House, 2003) and authored Analysis and Design of Autonomous Microwave Circuits (IEEE-Wiley, 2009).

Prof. Suárez is a member of the Technical Committees of the IEEE Microwave Theory and Techniques Society (IEEE MTT-S) International Microwave Symposium (IMS) and the European Microwave Conference. She was an IEEE Distinguished Microwave Lecturer from 2006 to 2008. She is a member of the Board of Directors of the European Microwave Association. She is the Editor-in-Chief of the International Journal of Microwave and Wireless Technologies (Cambridge University Press). She was the co-chair of IEEE Topical Conference on RF Power Amplifiers (PAWR) in 2014 and 2015. 